\title{
Enantioselective Synthesis of Nitroalkanes Bearing All-Carbon Quaternary Stereogenic Centers through Cu-Catalyzed Asymmetric Conjugate Additions
}

\author{
Jing Wu, Dawn M. Mampreian and Amir H. Hoveyda* \\ Department of Chemistry, Merkert Chemistry Center, Boston College \\ Chestnut Hill, Massachusetts 02467
}

\section{Supporting Information}

General. Infrared (IR) spectra are recorded on a Nicolet 210 spectrophotometer, $v_{\max }$ in $\mathrm{cm}^{-1}$. Bands are characterized as broad (br), strong (s), medium (m) or weak (w). ${ }^{1} \mathrm{H}$ NMR spectra are recorded on a Varian Unity INOVA $400(400 \mathrm{MHz})$ spectrometer. Chemical shifts are reported in ppm from tetramethylsilane with the solvent resonance resulting from incomplete deuteration as the internal standard $\left(\mathrm{CDCl}_{3}: \delta 7.26\right)$. Data are reported as follows: chemical shift, integration, multiplicity $(\mathrm{s}=$ singlet, $\mathrm{d}=$ doublet, $\mathrm{t}=$ triplet, $\mathrm{q}=$ quartet, $\mathrm{br}=$ broad, $\mathrm{m}=$ multiplet), coupling constants, and assignment. ${ }^{13} \mathrm{C}$ NMR spectra are recorded on a Varian Unity INOVA $400(100 \mathrm{MHz})$ spectrometer with complete proton decoupling. Chemical shifts are reported in ppm from tetramethylsilane with the solvent resonance as the internal standard $\left(\mathrm{CDCl}_{3}: \delta 77.16\right)$. Enantiomer ratios are determined by chiral GLC analysis; (Alltech Associates Chiraldex GTA column, 30m x $0.25 \mathrm{~mm}$ or Supelco Betadex 120 column, 30m x $0.25 \mathrm{~mm}$ ) or by analytical liquid chromatography (HPLC) on a Shimadzu chromatograph with a Chiralpack OD $(4.6 \times 250 \mathrm{~mm})$ chiral column by Chiral Technologies in comparison to authentic racemic materials. Racemic samples are made with a 1:1 mixture of D,D and L,L ligands. Elemental analyses are performed by Robertson Microlit Laboratories (Madison, New Jersey). High resolution mass spectrometry is performed by the University of Illinois Mass Spectrometry Laboratories (Urbana, Illinois) and at the Mass Spectrometry Facility, Boston College (on a Micromass LCT ESI-MS (positive mode)). Optical rotation values are recorded on a Rudolph Research Analytical Autopol IV Polarimeter.

Unless otherwise stated, all reactions are carried out under an inert atmosphere of $\mathrm{N}_{2}$ in flame dried glassware. Solvents are purified under a positive pressure of dry argon by a modified Advanced ChemTech purification system. Toluene is purified through $\mathrm{Cu}$ and alumina columns and $\mathrm{CH}_{2} \mathrm{Cl}_{2}, \mathrm{Et}_{2} \mathrm{O}$ and THF are purified by passage through two alumina columns. Diethylzinc (neat), dimethylzinc (1 $\mathrm{N}$ in toluene) and sodium nitrite are purchased from Aldrich and used without further purification. Dibutylzinc ( $1 \mathrm{~N}$ in heptane) is purchased from Fluka and used without further purification. Other alkylzincs are prepared based on reported methods. ${ }^{1 \mathrm{a}, \mathrm{b}}$ Copper (I) triflate (benzene complex, 2:1) is prepared by known methods. ${ }^{2}$ Nitroalkenes 1a-1c, 4 and $\mathbf{5}$ are synthesized according to a modified literature procedure. ${ }^{3}$ Stereochemistry of

1) a) Knochel, P.; Singer, P. R. Chem. Rev. 1993, 93, 2117-2188 and references cited therein.

2) a) Salomon, R. G.; Kochi, J. K. J. Am. Chem. Soc. 1973, 95, 1889-1897. b) Salomon, R. G.; Kochi, J. K. J. Am. Chem. Soc. 1973, 95, 3300-3310.

3) Ohta, H.; Kobayashi, N.; Ozaki, K. J. Org. Chem. 1989, 1802-1804. 
nitroalkenes has been ascertained previously. ${ }^{4}$ Peptide-based phosphine ligands are prepared as previously disclosed. ${ }^{5}$

Representative procedure for the synthesis of nitroalkenes. To a stirred mixture of acetic anhydride $(75.7 \mathrm{~g}, 742 \mathrm{mmol})$ and $69 \%$ aqueous nitric acid $(8.63 \mathrm{~g}, 94 \mathrm{mmol})$ is added 2(4-chlorophenyl)propene $(6.56 \mathrm{~g}, 43 \mathrm{mmol})$ at $-20{ }^{\circ} \mathrm{C}$ (dry ice/acetone bath) and the mixture is allowed to stir between -20 and $-10{ }^{\circ} \mathrm{C}$ for $10 \mathrm{~min}$. After the reaction is complete (as judged by TLC analysis), ice water $(25.0 \mathrm{~mL})$ is added and the mixture is allowed to stir at $0{ }^{\circ} \mathrm{C}$ for $15 \mathrm{~min}$ and then washed with diethyl ether $(3 \times 30 \mathrm{~mL})$. The combined organic layers are washed with saturated aqueous $\mathrm{NaHCO}_{3}$ and brine, dried over $\mathrm{MgSO}_{4}$, filtered and concentrated to afford a brown residue. Purification by silica gel chromatography (10:1 hexanes:ethyl acetate) affords 2acetoxy-1-nitro-2-(4-chlorophenyl)propane (9.23 g, $35.8 \mathrm{mmol}, 83 \%$ yield) as a yellow oil.

A solution of the nitroacetate, 2-acetoxy-1-nitro-2-(4-chlorophenyl)propane (3 g, 11.6 mmol) in $15 \%$ aqueous $\mathrm{NaOH}(60 \mathrm{~mL})$ and chloroform $(100 \mathrm{~mL})$ is allowed to stir at $22{ }^{\circ} \mathrm{C}$ for $24 \mathrm{~h}$. The mixture is washed with $\mathrm{CH}_{2} \mathrm{Cl}_{2}(3 \times 20 \mathrm{~mL})$. The organic layers are combined and washed with $10 \%$ aqueous $\mathrm{HCl}$, brine, dried over $\mathrm{MgSO}_{4}$, filtered and concentrated to afford a brown residue. Purification by silica gel chromatography (20:1 hexanes:ethyl acetate to elute product) affords (E)-1-nitro-2-(4-chlorophenyl)propene (1b, $1.66 \mathrm{~g}, 73 \%$ yield based on the nitro acetate used) as a yellow oil.

Representative procedure for the $\mathrm{Cu}$-catalyzed conjugate addition of dialkyl zinc reagents to nitroalkene. (CAUTION: $\mathrm{Et}_{2} \mathrm{Zn}$ is pyrophoric! Use extreme caution!) In a glovebox, a $13 \times 100 \mathrm{~mm}$ test tube is charged with $(\mathrm{CuOTf})_{2} \cdot \mathrm{C}_{6} \mathrm{H}_{6}(1.6 \mathrm{mg}, 3.2 \mu \mathrm{mol})$ and 3 (4.6 $\mathrm{mg}, 6.4 \mu \mathrm{mol})$, sealed with a septum. After the test tube is removed from the glovebox, toluene $(1.0 \mathrm{~mL})$ is added and the mixture is allowed to stir for $5 \mathrm{~min}$ at $22{ }^{\circ} \mathrm{C}$, and then cooled to -78 ${ }^{\circ} \mathrm{C}$ in a dry ice/acetone bath. $\mathrm{Et}_{2} \mathrm{Zn}(50.0 \mu \mathrm{L}, 0.49 \mathrm{mmol})$ is added to the mixture in a dropwise fashion and the resulting solution is allowed to stir at $-78{ }^{\circ} \mathrm{C}$ for $10 \mathrm{~min}$. A solution of $(E)-1$ nitro-2-phenylpropene (1a, $26 \mathrm{mg}, 0.16 \mathrm{mmol}$ ) in $1.0 \mathrm{~mL}$ of toluene is added by syringe and the reaction is allowed to stir at $-78{ }^{\circ} \mathrm{C}$. After $24 \mathrm{~h}$, the reaction is quenched at $-30{ }^{\circ} \mathrm{C}$ by the dropwise addition of a saturated aqueous solution of $\mathrm{NH}_{4} \mathrm{Cl}(5.0 \mathrm{~mL})$. The mixture is washed with $\mathrm{Et}_{2} \mathrm{O}(2 \times 2.0 \mathrm{~mL})$. The combined organic layers are passed through a short plug of silica gel eluting with $\mathrm{Et}_{2} \mathrm{O}$. Volatiles are removed under reduced pressure and the toluene solution is purified by silica gel chromatography (100\% pentane to elute toluene followed by $20: 1$ pentane: $\mathrm{Et}_{2} \mathrm{O}$ to elute product) to afford a colorless oil (27 $\mathrm{mg}, 0.14 \mathrm{mmol}, 87 \%$ yield).

Representative procedure for the transformation of nitroalkanes to corresponding carboxylic acids. A solution of 1-nitro-2-methyl-2-(4-chlorophenyl)butane (7, $19 \mathrm{mg}, 0.083$ mmol), sodium nitrite $(17.3 \mathrm{mg}, 0.25 \mathrm{mmol})$ and acetic acid $(50.1 \mathrm{mg}, 0.83 \mathrm{mmol})$ in dimethyl sulfoxide $(250 \mu \mathrm{L})$ is allowed to stir at $35^{\circ} \mathrm{C}$ for $48 \mathrm{~h}$. After acidification with a $10 \%$ aqueous solution of hydrochloric acid $(2 \mathrm{~mL})$, the product is washed with diethyl ether $(3 \times 5 \mathrm{~mL})$. The combined organic layers are dried over $\mathrm{MgSO}_{4}$, filtered and concentrated to afford a yellow residue. Purification by silica gel chromatography (10:1 pentane: $\mathrm{Et}_{2} \mathrm{O}$ to elute product) delivers 2-methyl-2-(4-chlorophenyl)butanoic acid (19) as a colorless oil (14.3 $\mathrm{mg}, 0.067 \mathrm{mmol}, 81 \%$ yield).

Representative procedure for the transformation of nitroalkanes to corresponding primary amines. 1-Nitro-2-methyl-2-(4-chlorophenyl)butane (7, $80 \mathrm{mg}, 0.35 \mathrm{mmol}), \operatorname{Pd}(\mathrm{C})$

4) Czekelius, C.; Carreira, E. M. Angew. Chem. Int. Ed. 2003, 42, 4793-4795.

5) Mampreian, D. M.; Hoveyda, A. H. Org. Lett. 2004, 6, 2829-2832. 
$(10 \%, 8.0 \mathrm{mg})$ and $\mathrm{MeOH}(2 \mathrm{~mL})$ are charged into a autoclave equipped with a magnetic stirring bar. Hydrogen is initially introduced into the autoclave at a pressure of 300 psi before being reduced to $10-20$ psi by carefully releasing the stop valve. After this procedure is repeated three times, the vessel is pressurized to $600 \mathrm{psi}$. The reaction mixture is allowed to stir at $22{ }^{\circ} \mathrm{C}$ for 40 $\mathrm{h}$. After releasing the hydrogen gas and filtering off the solid catalyst, the solution is evaporated to dryness. The residue is purified by silica gel chromatography ( $1: 1$ pentane:Et ${ }_{2} \mathrm{O}$ to elute product) to afford 1-amino-2-methyl-2-(4-chlorophenyl)butane as a colorless oil (43.6 mg, 0.22 mmol, 63\% yield).

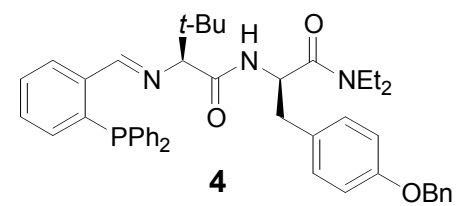

2-(Diphenylphosphino)-L-Tle-D-Tyr(OBn)-NEt $\mathbf{2}_{\mathbf{2}}$ (4). M.p. $=63-68$ ${ }^{\circ} \mathrm{C}$. IR (neat): 3396 (w), 3276 (br), 3056 (m), 2974 (m), 2930 (m), $2867(\mathrm{w}), 1954(\mathrm{w}), 1885(\mathrm{w}), 1810(\mathrm{w}), 1646(\mathrm{~s}), 1583(\mathrm{w}), 1508$ (s), $1464(\mathrm{~s}), 1438(\mathrm{~s}), 1382(\mathrm{~m}), 1369(\mathrm{w}), 1269$ (s), 1243 (s), 1180 (m), $1143(\mathrm{w}), 1105(\mathrm{w}), 1029(\mathrm{~m}), 746(\mathrm{~s}), 620(\mathrm{~s}) \mathrm{cm}^{-1} \cdot{ }^{1} \mathrm{H}$ NMR $\left(\mathrm{CDCl}_{3}, 400 \mathrm{MHz}\right): \delta 8.59(1 \mathrm{H}, \mathrm{d}, J=4.4 \mathrm{~Hz}, \mathrm{~N}=\mathrm{CH}), 7.93(1 \mathrm{H}, \mathrm{ddd}, J=6.8,4.0,1.2 \mathrm{~Hz}$, $\operatorname{ArH}), 7.59(1 \mathrm{H}, \mathrm{d}, J=7.2 \mathrm{~Hz}, \operatorname{ArH}), 7.45-7.22(17 \mathrm{H}, \mathrm{m}, \operatorname{ArH}$ and NH), 7.04-7.02 (2H, m, ArH), $6.90(1 \mathrm{H}, \mathrm{ddd}, J=7.6,4.4,1.2 \mathrm{~Hz}, \operatorname{ArH}), 6.73-6.71(2 \mathrm{H}, \mathrm{m}, \operatorname{ArH}), 5.04-4.97(1 \mathrm{H}, \mathrm{m}$, NHCHCO), 4.93 (2H, s, $\left.\mathrm{PhCH}_{2} \mathrm{O}\right), 3.39-3.21$ (4H, m, $\mathrm{CH}_{3} \mathrm{CHHNCH}_{2} \mathrm{CH}_{3}$ and $\left.\mathrm{NCH} t-\mathrm{Bu}\right), 3.09-$ $2.97\left(2 \mathrm{H}, \mathrm{m}, \mathrm{CH}_{3} \mathrm{CHHNCH}_{2} \mathrm{CH}_{3}\right.$ and $\left.\mathrm{CHCHHAr}\right), 2.82(1 \mathrm{H}, \mathrm{dd}, J=13.2,6.0 \mathrm{~Hz}, \mathrm{CHCHHAr})$, $1.03\left(3 \mathrm{H}, \mathrm{t}, J=7.2 \mathrm{~Hz}, \mathrm{NCH}_{2} \mathrm{CH}_{3}\right), 0.97\left(3 \mathrm{H}, \mathrm{t}, J=7.2 \mathrm{~Hz}, \mathrm{NCH}_{2} \mathrm{CH}_{3}\right), 0.68(9 \mathrm{H}, \mathrm{s}, t-\mathrm{Bu}) .{ }^{13} \mathrm{C}$ NMR $\left(\mathrm{CDCl}_{3}, 100 \mathrm{MHz}\right): \delta 170.3,170.2,160.6,160.4,157.5,138.7,138.5,137.8,137.6,137.1$, 137.0, 136.9, 136.2, 136.1, 134.5, 134.3, 133.9, 133.7, 130.6, 129.4, 129.3, 129.0, 128.9, 128.7, 128.6, 128.5, 127.9, 127.5, 114.7, 84.5, 70.0, 50.0, 41.9, 40.7, 38.7, 35.3, 27.2, 14.8, 13.1. HRMS Calcd. for $\mathrm{C}_{45} \mathrm{H}_{49} \mathrm{~N}_{3} \mathrm{O}_{3} \mathrm{PH}\left[\mathrm{M}+\mathrm{H}^{+}\right]$: 712.3668. Found: 712.3649. Anal. Calcd for $\mathrm{C}_{45} \mathrm{H}_{49} \mathrm{~N}_{3} \mathrm{O}_{3} \mathrm{P}$ : C, 75.92; H, 7.08; N, 5.90. Found: C, 75.85; H, 7.24; N, 5.90. $[\alpha]_{\mathrm{D}}{ }^{20}+13.2^{\circ}(c$ $\left.=1.22, \mathrm{CHCl}_{3}\right)$.

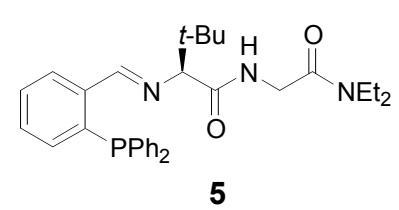

2-(Diphenylphosphino)-L-Tle-Gly-NEt 2 (5). M.p. $=68-72{ }^{\circ} \mathrm{C} . \quad$ IR (neat): 3383 (br), $3050(\mathrm{~m}), 2967(\mathrm{~m}), 2924(\mathrm{~m}), 2873(\mathrm{~m}), 2320(\mathrm{w})$, 1967 (w), 1652 (s), 1507 (m), 1483 (s), 1464 (m), 1438 (s), 1394 (m), 1375 (m), 1249 (m), 1313 (w), 1269 (m), $1224(\mathrm{w}), 1155(\mathrm{w}), 1123(\mathrm{w})$, 1073 (w), 1029 (w), 746 (s), 696 (s) cm ${ }^{-1}$. ${ }^{1} \mathrm{H} \mathrm{NMR}\left(\mathrm{CDCl}_{3}, 400 \mathrm{MHz}\right)$ : $\delta 8.73(1 \mathrm{H}, \mathrm{d}, J=5.2 \mathrm{~Hz}, \mathrm{~N}=\mathrm{CH}), 8.13(1 \mathrm{H}, \mathrm{ddd}, J=7.6,4.0,1.2 \mathrm{~Hz}, \operatorname{ArH}), 7.48-7.42(2 \mathrm{H}, \mathrm{m}$, $\operatorname{ArH}), 7.36-7.22(11 \mathrm{H}, \mathrm{m}, \operatorname{ArH}$ and NH), $6.87(1 \mathrm{H}, \mathrm{ddd}, J=7.6,4.4,1.2 \mathrm{~Hz}, \mathrm{ArH}), 4.06(1 \mathrm{H}, \mathrm{dd}$, $J=17.2,4.8 \mathrm{~Hz}, \mathrm{NCHHCO}), 3.94(1 \mathrm{H}, \mathrm{dd}, J=17.2,4.4 \mathrm{~Hz}, \mathrm{NCHHCO}), 3.44-3.38$ (3H, m, $\mathrm{NCH} t$ - $\mathrm{Bu}$ and $\left.\mathrm{NCH}_{2} \mathrm{CH}_{3}\right), 3.27\left(2 \mathrm{H}, \mathrm{dq}, J=7.2,2.4 \mathrm{~Hz}, \mathrm{NCH}_{2} \mathrm{CH}_{3}\right), 1.19(3 \mathrm{H}, \mathrm{t}, J=7.2 \mathrm{~Hz}$, $\left.\mathrm{NCH}_{2} \mathrm{CH}_{3}\right), 1.14\left(3 \mathrm{H}, \mathrm{t}, J=6.8 \mathrm{~Hz}, \mathrm{NCH}_{2} \mathrm{CH}_{3}\right), 0.85(9 \mathrm{H}, \mathrm{s}, t-\mathrm{Bu}) .{ }^{13} \mathrm{C} \mathrm{NMR}\left(\mathrm{CDCl}_{3}, 100\right.$ $\mathrm{MHz}): \delta 171.7,167.2,160.7,160.5,138.9,137.9,136.8,136.4,134.3,134.1,133.6,130.7$, 129.2, 128.9, 128.8, 128.7, 128.6, 84.3, 41.1, 40.9, 40.4, 35.3, 27.2, 14.2, 13.1. HRMS Calcd. for $\mathrm{C}_{31} \mathrm{H}_{38} \mathrm{~N}_{3} \mathrm{O}_{2} \mathrm{PH}\left[\mathrm{M}+\mathrm{H}^{+}\right]$: 516.2780. Found: 516.2783. Anal. Calcd for $\mathrm{C}_{31} \mathrm{H}_{38} \mathrm{~N}_{3} \mathrm{O}_{2} \mathrm{P}$ : C, 72.21; H, 7.43; N, 8.15. Found: C, 71.98; H, 7.04; N, 7.81. $[\alpha]_{\mathrm{D}}{ }^{20}-27.86^{\circ}\left(c=0.787, \mathrm{CHCl}_{3}\right)$.

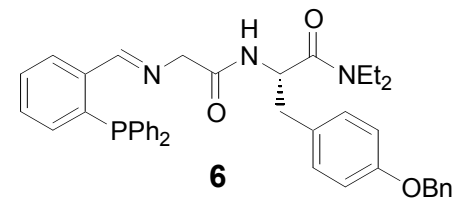

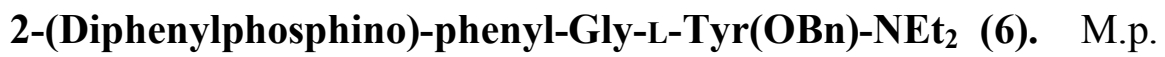
58-62 ${ }^{\circ} \mathrm{C}$. IR (neat): 3377 (w), 3283 (br), $3056(\mathrm{~m}), 2980(\mathrm{~m}), 2930$ (m), $2873(\mathrm{w}), 1960(\mathrm{w}), 1885(\mathrm{w}), 1810(\mathrm{w}), 1678(\mathrm{~s}), 1640(\mathrm{~s})$, 1583 (m), 1508 (s), 1463 (m), 1438 (m), 1382 (m), 1243 (s), 1180 
(m), 1142 (w), 1099 (w), 1067 (w), 1029 (m), 740 (s), 702 (s) cm ${ }^{-1} \cdot{ }^{1} \mathrm{H} \mathrm{NMR}_{\text {(CDCl }}, 400$ $\mathrm{MHz}): \delta 8.84(1 \mathrm{H}, \mathrm{d}, J=4.8 \mathrm{~Hz}, \mathrm{~N}=\mathrm{CH}), 8.00(1 \mathrm{H}, \mathrm{ddd}, J=7.6,4.0,1.6 \mathrm{~Hz}, \operatorname{ArH}), 7.80(1 \mathrm{H}, \mathrm{d}$, $J=8.4 \mathrm{~Hz}, \operatorname{ArH}), 7.44-7.21(17 \mathrm{H}, \mathrm{m}, \operatorname{ArH}$ and NH), 7.10-7.07 (2H, m, ArH), $6.92(1 \mathrm{H}, \mathrm{ddd}, J=$ 7.6, 4.8, $1.2 \mathrm{~Hz}, \mathrm{ArH}), 6.83-6.79(2 \mathrm{H}, \mathrm{m}, \mathrm{ArH}), 5.09-5.03(1 \mathrm{H}, \mathrm{m}, \mathrm{NHCHCO}), 4.99$ (2H, s, $\left.\mathrm{PhCH}_{2} \mathrm{O}\right), 4.15-4.04\left(2 \mathrm{H}, \mathrm{m}, \mathrm{C}=\mathrm{NCH}_{2} \mathrm{CO}\right), 3.53\left(1 \mathrm{H}, \mathrm{dq}, J=13.6,7.2 \mathrm{~Hz}, \mathrm{CH}_{3} \mathrm{CHHNCH}_{2} \mathrm{CH}_{3}\right)$, 3.08-2.95 $\left(4 \mathrm{H}, \mathrm{m}, \mathrm{CH}_{3} \mathrm{CHHNCH}_{2} \mathrm{CH}_{3}\right.$ and $\left.\mathrm{CHCHHAr}\right), 2.91(1 \mathrm{H}, \mathrm{dd}, J=13.2,5.6 \mathrm{~Hz}$, CHCHHAr), $1.01\left(3 \mathrm{H}, \mathrm{t}, J=7.2 \mathrm{~Hz}, \mathrm{NCH}_{2} \mathrm{CH}_{3}\right), 0.96\left(3 \mathrm{H}, \mathrm{t}, J=7.2 \mathrm{~Hz}, \mathrm{NCH}_{2} \mathrm{CH}_{3}\right) .{ }^{13} \mathrm{C} \mathrm{NMR}$ $\left(\mathrm{CDCl}_{3}, 100 \mathrm{MHz}\right): \delta 170.4,169.4,162.4,162.2,157.8,138.8,138.6,138.2,138.0,137.2$, $136.6,136.5,134.2,134.0,133.9,131.0,130.8,129.2,129.1,129.0,128.8,128.7,128.6,128.0$, 127.6, 115.0, 70.2, 63.0, 50.1, 41.8, 40.6, 39.3, 14.4, 13.0. HRMS Calcd for $\mathrm{C}_{41} \mathrm{H}_{42} \mathrm{~N}_{3} \mathrm{O}_{3} \mathrm{PH}$ $\left[\mathrm{M}+\mathrm{H}^{+}\right]:$656.3042. Found: 656.3047. Anal. Calcd for $\mathrm{C}_{41} \mathrm{H}_{42} \mathrm{~N}_{3} \mathrm{O}_{3} \mathrm{P}: \mathrm{C}, 75.09 ; \mathrm{H}, 6.46 ; \mathrm{N}$, 6.41. Found: C, 74.87; $\mathrm{H}, 6.60 ; \mathrm{N}, 6.18 .[\alpha]_{\mathrm{D}}{ }^{20}+11.93^{\circ}\left(c=0.487, \mathrm{CHCl}_{3}\right)$.

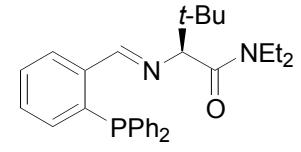

7

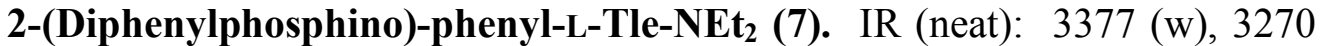
(w), $3056(\mathrm{w}), 2968(\mathrm{~m}), 2899(\mathrm{w}), 2867(\mathrm{w}), 1698(\mathrm{~m}), 1640(\mathrm{~s}), 1501(\mathrm{~m})$, 1476 (m), 1464 (m), 1438 (m), 1369 (m), 1269 (m), 1218 (m), 1143 (w), 1099 (w), 1073 (w), 1029 (w), 746 (s), $696(\mathrm{~m}) \mathrm{cm}^{-1}$. ${ }^{1} \mathrm{H}$ NMR (CDCl 3 , $\left.400 \mathrm{MHz}\right)$ : $\delta 8.72(1 \mathrm{H}, \mathrm{d}, J=4.8 \mathrm{~Hz}, \mathrm{~N}=\mathrm{CH}), 8.05(1 \mathrm{H}, \mathrm{ddd}, J=7.6,3.6,0.8 \mathrm{~Hz}, \operatorname{ArH}), 7.59(1 \mathrm{H}, \mathrm{d}, J=9.6$ $\mathrm{Hz}, \operatorname{ArH}), 7.45$ (1H, ddd, $J=8.0,8.0,0.4 \mathrm{~Hz}, \operatorname{ArH}), 7.33-7.20$ (10H, m, ArH), 6.86 (1H, ddd, $J$ $=7.6,4.8,0.8 \mathrm{~Hz}, \mathrm{ArH}), 3.68-3.54\left(2 \mathrm{H}, \mathrm{m}, \mathrm{NCH}_{2} \mathrm{CH}_{3}\right), 3.38(1 \mathrm{H}, \mathrm{s}, \mathrm{NCH} t-\mathrm{Bu}), 3.27(1 \mathrm{H}, \mathrm{dq}, J$ $\left.=14.4,7.2 \mathrm{~Hz}, \mathrm{NCHHCH}_{3}\right), 2.98\left(\mathrm{dq}, J=14.4,7.2 \mathrm{~Hz}, \mathrm{NCHHCH}_{3}\right), 1.17(3 \mathrm{H}, \mathrm{t}, J=6.8 \mathrm{~Hz}$, $\left.\mathrm{NCH}_{2} \mathrm{CH}_{3}\right), 1.04\left(3 \mathrm{H}, \mathrm{t}, J=7.2 \mathrm{~Hz}, \mathrm{NCH}_{2} \mathrm{CH}_{3}\right), 0.78(9 \mathrm{H}, \mathrm{s}, t-\mathrm{Bu}) .{ }^{13} \mathrm{C} \mathrm{NMR}\left(\mathrm{CDCl}_{3}, 100\right.$ $\mathrm{MHz}): \delta 171.3,170.4,161.1,160.8,139.0,138.9,137.9,137.7,136.7,136.6,136.3,134.6$, $134.4,134.0,133.9,133.5,130.7,129.3,129.1,128.9,128.8,128.7,128.6,84.3,54.1,42.9,40.4$, $35.7,35.2,27.2,14.6,13.0 .[\alpha]_{\mathrm{D}}{ }^{20}+2.0^{\circ}\left(c=2.0, \mathrm{CHCl}_{3}\right)$.

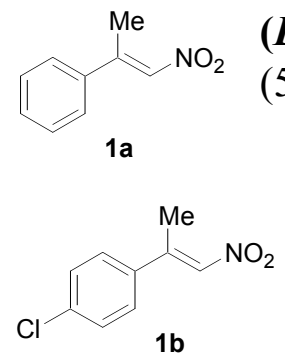

(E)-1-Nitro-2-phenyl-1-propene (1a). ${ }^{1} \mathrm{H}$ NMR $\left(\mathrm{CDCl}_{3}, 400 \mathrm{MHz}\right): \delta$ 7.45-7.37 $(5 \mathrm{H}, \mathrm{m}, \mathrm{ArH}), 7.32\left(1 \mathrm{H}, \mathrm{s}, \mathrm{CHNO}_{2}\right), 2.63\left(3 \mathrm{H}, \mathrm{s}, \operatorname{ArCCH}_{3}\right)$.

(E)-1-Nitro-2-(4-chlorophenyl)-1-propene (1b). ${ }^{1} \mathrm{H} \quad \mathrm{NMR}\left(\mathrm{CDCl}_{3}, 400\right.$ $\mathrm{MHz}): \delta 7.43-7.38(4 \mathrm{H}, \mathrm{m}, \mathrm{ArH}), 7.28\left(1 \mathrm{H}, \mathrm{q}, J=1.6 \mathrm{~Hz}, \mathrm{CHNO}_{2}\right), 2.62(3 \mathrm{H}$, $\left.\mathrm{d}, J=1.6 \mathrm{~Hz}, \mathrm{CH}_{3}\right) \cdot{ }^{13} \mathrm{C} \mathrm{NMR}\left(\mathrm{CDCl}_{3}, 100 \mathrm{MHz}\right): \delta 148.6,136.8,136.6$, $129.5,128.4,128.3,18.6$.

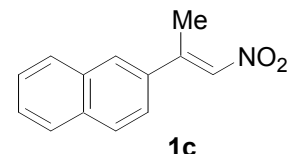

(E)-1-Nitro-2-(2'-napthyl)-1-propene (1c). IR (neat): 3100 (w), 3049 (w), 2962 (w), $2930(\mathrm{~m}), 2861$ (w), $1621(\mathrm{~m}), 1514(\mathrm{~s}), 1470(\mathrm{w}), 1439$ (w), 1388 (m), 1344 (s), $1281(\mathrm{w}), 1231(\mathrm{w}), 1199(\mathrm{w}), 1143(\mathrm{w}), 1029(\mathrm{w}), 960(\mathrm{w})$, $828(\mathrm{~m}), 759(\mathrm{~m}), 708(\mathrm{w}) \mathrm{cm}^{-1} .{ }^{1} \mathrm{H} \mathrm{NMR}\left(\mathrm{CDCl}_{3}, 400 \mathrm{MHz}\right): \delta 7.96(1 \mathrm{H}, \mathrm{d}$, $J=1.6 \mathrm{~Hz}, \operatorname{ArH}), 7.91-7.86(3 \mathrm{H}, \mathrm{m}, \operatorname{ArH}), 7.58-7.52(3 \mathrm{H}, \mathrm{m}, \operatorname{ArH}), 7.46\left(1 \mathrm{H}, \mathrm{m}, \mathrm{CHNO}_{2}\right), 2.76$ $\left(3 \mathrm{H}, \mathrm{d}, J=1.6 \mathrm{~Hz}, \mathrm{CH}_{3}\right) .{ }^{13} \mathrm{C} \mathrm{NMR}\left(\mathrm{CDCl}_{3}, 100 \mathrm{MHz}\right): \delta 150.0,136.8,135.6,134.1,133.2$, 129.1, 128.7, 127.9, 127.7, 127.20, 127.18, 123.8, 18.7. HRMS Calcd for $\mathrm{C}_{13} \mathrm{H}_{11} \mathrm{NO}_{2}$ : 213.0790. Found: 213.0788 .

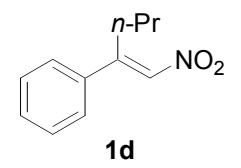

(E)-1-Nitro-2-phenyl-1-pentene (1d). ${ }^{1} \mathrm{H} \mathrm{NMR}\left(\mathrm{CDCl}_{3}, 400 \mathrm{MHz}\right): \delta$ 7.44-7.39 (5H, m, ArH), $7.19\left(1 \mathrm{H}, \mathrm{s}, \mathrm{CHNO}_{2}\right), 3.06-3.02\left(2 \mathrm{H}, \mathrm{m}, \mathrm{CH}_{2} \mathrm{CH}_{2} \mathrm{CH}_{3}\right), 1.57-1.47$ 


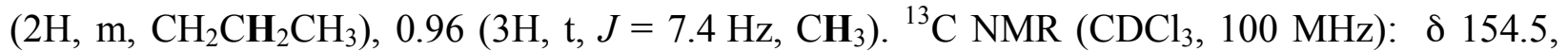
$137.5,136.3,130.3,129.2,127.2,33.1,21.9,14.2$.

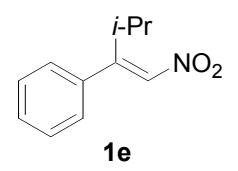

$\mathrm{Ne}^{\mathrm{Me}}$

$(S)-2 a$

(E)-1-Nitro-3-methyl-2-phenyl-1-butene (1e). ${ }^{1} \mathrm{H} \mathrm{NMR}\left(\mathrm{CDCl}_{3}, 400 \mathrm{MHz}\right): \delta$ 7.41-7.36 (3H, m, ArH), 7.24-7.21 (3H, m, ArH), $6.88\left(1 \mathrm{H}, \mathrm{s}, \mathrm{CHNO}_{2}\right), 3.96(1 \mathrm{H}$, qq, $\left.J=6.8,6.8 \mathrm{~Hz}, \mathrm{CH}\left(\mathrm{CH}_{3}\right)_{2}\right), 1.13\left(6 \mathrm{H}, \mathrm{d}, J=6.8 \mathrm{~Hz}, \mathrm{CH}\left(\mathrm{CH}_{3}\right)_{2}\right) .{ }^{13} \mathrm{C} \mathrm{NMR}$ $\left(\mathrm{CDCl}_{3}, 100 \mathrm{MHz}\right): \delta 159.2,136.4,135.8,128.9,128.4,128.0,29.4,20.9$.

(S)-1-Nitro-2-methyl-2-phenylbutane (2a). IR (neat): 3106 (w), 3074 (w), 2974 (s), $2936(\mathrm{w}), 2892(\mathrm{w}), 1552(\mathrm{~s}), 1501(\mathrm{~m}), 1451(\mathrm{~m}), 1381(\mathrm{~s}), 1331(\mathrm{w}), 1250$ (w), $1180(\mathrm{w}), 1048(\mathrm{w}), 1004(\mathrm{w}), 916(\mathrm{w}), 765(\mathrm{~s}), 708(\mathrm{~s}), 645(\mathrm{~m}), 557(\mathrm{~m})$ $\mathrm{cm}^{-1} .{ }^{1} \mathrm{H} \mathrm{NMR}\left(\mathrm{CDCl}_{3}, 400 \mathrm{MHz}\right): \delta 7.37-7.23(5 \mathrm{H}, \mathrm{m}, \mathrm{ArH}), 4.59(\mathrm{H}, \mathrm{d}, J=$ $\left.10.8 \mathrm{~Hz}, \mathrm{CHHNO}_{2}\right), 4.54\left(\mathrm{H}, \mathrm{d}, J=10.8 \mathrm{~Hz}, \mathrm{CHHNO}_{2}\right), 1.95(1 \mathrm{H}, \mathrm{dq}, J=14.8,7.2 \mathrm{~Hz}$, $\left.\mathrm{CHHCH}_{3}\right), 1.74\left(1 \mathrm{H}, \mathrm{dq}, J=14.8,7.2 \mathrm{~Hz}, \mathrm{CHHCH}_{3}\right), 1.52(3 \mathrm{H}, \mathrm{s}, \operatorname{ArCCH} 3), 0.73(3 \mathrm{H}, \mathrm{t}, J=7.4$ $\left.\mathrm{Hz}, \mathrm{CH}_{2} \mathrm{CH}_{3}\right) .{ }^{13} \mathrm{C} \mathrm{NMR}\left(\mathrm{CDCl}_{3}, 100 \mathrm{MHz}\right): \delta 142.2,128.7,127.1,126.3,86.3,42.8,32.5,22.0$, 8.4. HRMS Calcd for $\mathrm{C}_{11} \mathrm{H}_{15} \mathrm{NO}_{2}$ : 193.1103. Found: 193.1103. Anal. Calcd for $\mathrm{C}_{11} \mathrm{H}_{15} \mathrm{NO}_{2}$ : C, 68.37; H, 7.82; N, 7.25. Found: C, 68.02; H, 7.61; N, 7.19. $[\alpha]_{\mathrm{D}}{ }^{20}-31.6^{\circ}\left(c=1.50, \mathrm{CHCl}_{3}\right)$ for a sample that is $94 \%$ ee.

Optical purity is established by chiral GLC analysis (Chiraldex GTA, $110{ }^{\circ} \mathrm{C}, 20 \mathrm{psi}$ ); chromatograms are illustrated below for a $94 \%$ ee sample:
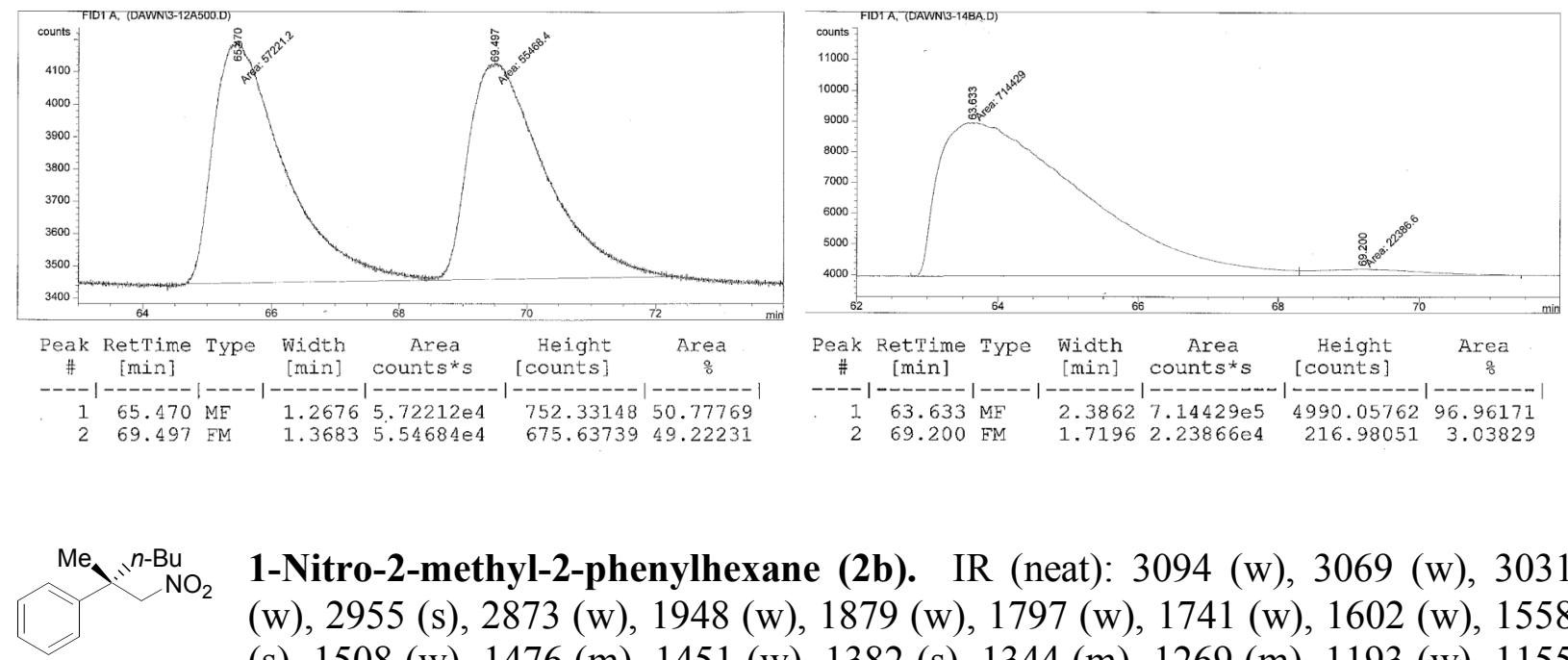

1-Nitro-2-methyl-2-phenylhexane (2b). IR (neat): 3094 (w), 3069 (w), 3031 (w), $2955(\mathrm{~s}), 2873(\mathrm{w}), 1948(\mathrm{w}), 1879(\mathrm{w}), 1797(\mathrm{w}), 1741(\mathrm{w}), 1602(\mathrm{w}), 1558$

2b

(s), $1508(\mathrm{w}), 1476(\mathrm{~m}), 1451(\mathrm{w}), 1382(\mathrm{~s}), 1344(\mathrm{~m}), 1269(\mathrm{~m}), 1193(\mathrm{w}), 1155$ (m), 1080 (m), 1042 (s), 973 (w), 910 (m), 790 (s), 696 (s), 658 (s), 570 (m) cm ${ }^{-1}$. ${ }^{1} \mathrm{H}$ NMR $\left(\mathrm{CDCl}_{3}, 400 \mathrm{MHz}\right): \delta$ 7.37-7.23 $(5 \mathrm{H}, \mathrm{m}, \mathrm{ArH}), 4.58\left(1 \mathrm{H}, \mathrm{d}, J=10.4 \mathrm{~Hz}, \mathrm{CHHNO}_{2}\right), 4.54(1 \mathrm{H}$, $\left.\mathrm{d}, J=10.4 \mathrm{~Hz}, \mathrm{CHHNO}_{2}\right), 1.88\left(1 \mathrm{H}, \mathrm{dt}, J=12.8,4.0 \mathrm{~Hz}, \mathrm{CHH}\left(\mathrm{CH}_{2}\right)_{2} \mathrm{CH}_{3}\right), 1.68(1 \mathrm{H}, \mathrm{dt}, J=$ 12.8, $\left.4.4 \mathrm{~Hz}, \mathrm{CHH}\left(\mathrm{CH}_{2}\right)_{2} \mathrm{CH}_{3}\right), 1.53\left(3 \mathrm{H}, \mathrm{s}, \mathrm{ArCCH}_{3}\right), 1.30-0.94\left(4 \mathrm{H}, \mathrm{m}, \mathrm{CH}_{2}\left(\mathrm{CH}_{2}\right)_{2} \mathrm{CH}_{3}\right), 0.82$ $\left(3 \mathrm{H}, \mathrm{t}, J=7.2 \mathrm{~Hz},\left(\mathrm{CH}_{2}\right)_{3} \mathrm{CH}_{3}\right) .{ }^{13} \mathrm{C} \mathrm{NMR}\left(\mathrm{CDCl}_{3}, 100 \mathrm{MHz}\right): \delta 142.6,128.7,127.1,126.2$, 86.6, 42.5, 39.6, 26.1, 23.2, 22.6, 14.0. HRMS Calcd for $\mathrm{C}_{13} \mathrm{H}_{19} \mathrm{NO}_{2}$ : 221.1416. Found: 221.1416. Anal. Calcd for $\mathrm{C}_{13} \mathrm{H}_{19} \mathrm{NO}_{2}$ : C, 70.56; H, 8.65; N, 6.33. Found: C, 70.48; H, 8.83; N, 6.33. $[\alpha]_{\mathrm{D}}{ }^{20}-30.5^{\circ}\left(c=1.70, \mathrm{CHCl}_{3}\right)$ for a sample that is $93 \%$ ee.

Optical purity is established by chiral GLC analysis (Chiraldex GTA, $140{ }^{\circ} \mathrm{C}, 15 \mathrm{psi}$ ); chromatograms are illustrated below for a $93 \%$ ee sample: 

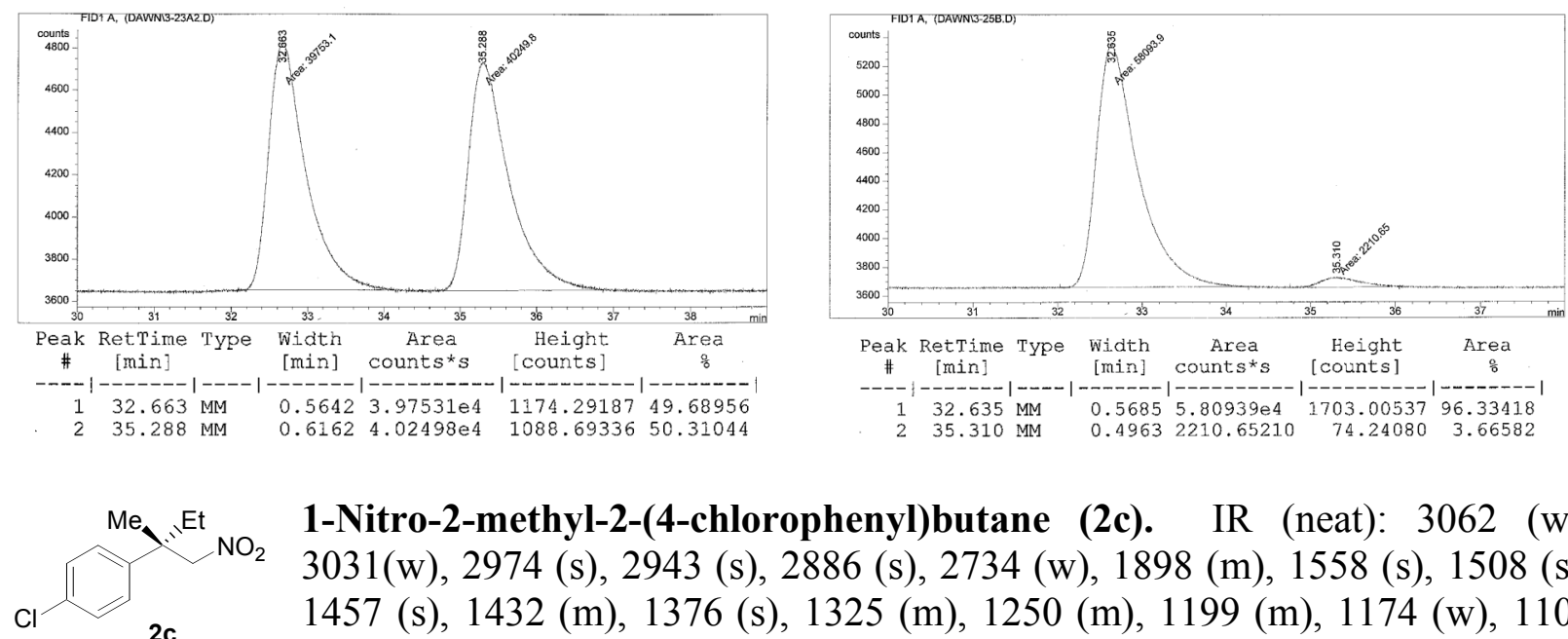

1-Nitro-2-methyl-2-(4-chlorophenyl)butane (2c). IR (neat): 3062 (w), 3031(w), 2974 (s), 2943 (s), 2886 (s), 2734 (w), 1898 (m), 1558 (s), 1508 (s), $1457(\mathrm{~s}), 1432(\mathrm{~m}), 1376(\mathrm{~s}), 1325(\mathrm{~m}), 1250(\mathrm{~m}), 1199(\mathrm{~m}), 1174(\mathrm{w}), 1105$ (s), $1010(\mathrm{~s}), 903(\mathrm{~m}), 821(\mathrm{~s}), 790(\mathrm{~m}), 765$ (m), 740 (s), 721 (s), $664(\mathrm{~m}), 551$ (m) $\mathrm{cm}^{-1} .{ }^{1} \mathrm{H}$ NMR $\left(\mathrm{CDCl}_{3}, 400 \mathrm{MHz}\right): \delta 7.32-7.30(2 \mathrm{H}, \mathrm{m}, \operatorname{ArH}), 7.24-7.21(2 \mathrm{H}, \mathrm{m}, \operatorname{ArH})$, $4.57\left(1 \mathrm{H}, \mathrm{d}, J=10.8 \mathrm{~Hz}, \mathrm{CHHNO}_{2}\right), 4.50\left(1 \mathrm{H}, \mathrm{d}, J=10.8 \mathrm{~Hz}, \mathrm{CHHNO}_{2}\right), 1.90(1 \mathrm{H}, \mathrm{dq}, J=$ 13.6, 7.6 Hz, $\left.\mathrm{CHHCH}_{3}\right), 1.71\left(1 \mathrm{H}, \mathrm{dq}, J=14.0,7.6 \mathrm{~Hz}, \mathrm{CHHCH}_{3}\right), 1.50\left(3 \mathrm{H}, \mathrm{s}, \mathrm{CCH}_{3}\right), 0.73$ $\left(3 \mathrm{H}, \mathrm{t}, J=7.6 \mathrm{~Hz}, \mathrm{CH}_{2} \mathrm{CH}_{3}\right) .{ }^{13} \mathrm{C} \mathrm{NMR}\left(\mathrm{CDCl}_{3}, 100 \mathrm{MHz}\right): \delta 140.7,133.0,128.9,127.8,86.1$, 42.6, 32.6, 22.0, 8.3. HRMS Calcd for $\mathrm{C}_{11} \mathrm{H}_{14} \mathrm{ClNO}_{2}$ : 227.0713. Found: 227.0717. Anal. Calcd for $\mathrm{C}_{11} \mathrm{H}_{14} \mathrm{ClNO}_{2}$ : C, 58.03; H, 6.20; N, 6.15. Found: C, 58.16; H, 6.32; N, 6.19. $[\alpha]_{\mathrm{D}}{ }^{20}{ }_{-} 45.5^{\circ}$ $\left(c=0.840, \mathrm{CHCl}_{3}\right)$ for a sample that is $98 \%$ ee.

Optical purity is established by chiral HPLC analysis (Chiral Technologies Chiralpak OD, $254 \mathrm{~nm}, 98: 2$ hexanes $/ i-\mathrm{PrOH}, 0.3 \mathrm{~mL} / \mathrm{min}$ ); chromatograms are illustrated below for a 98\% ee sample:
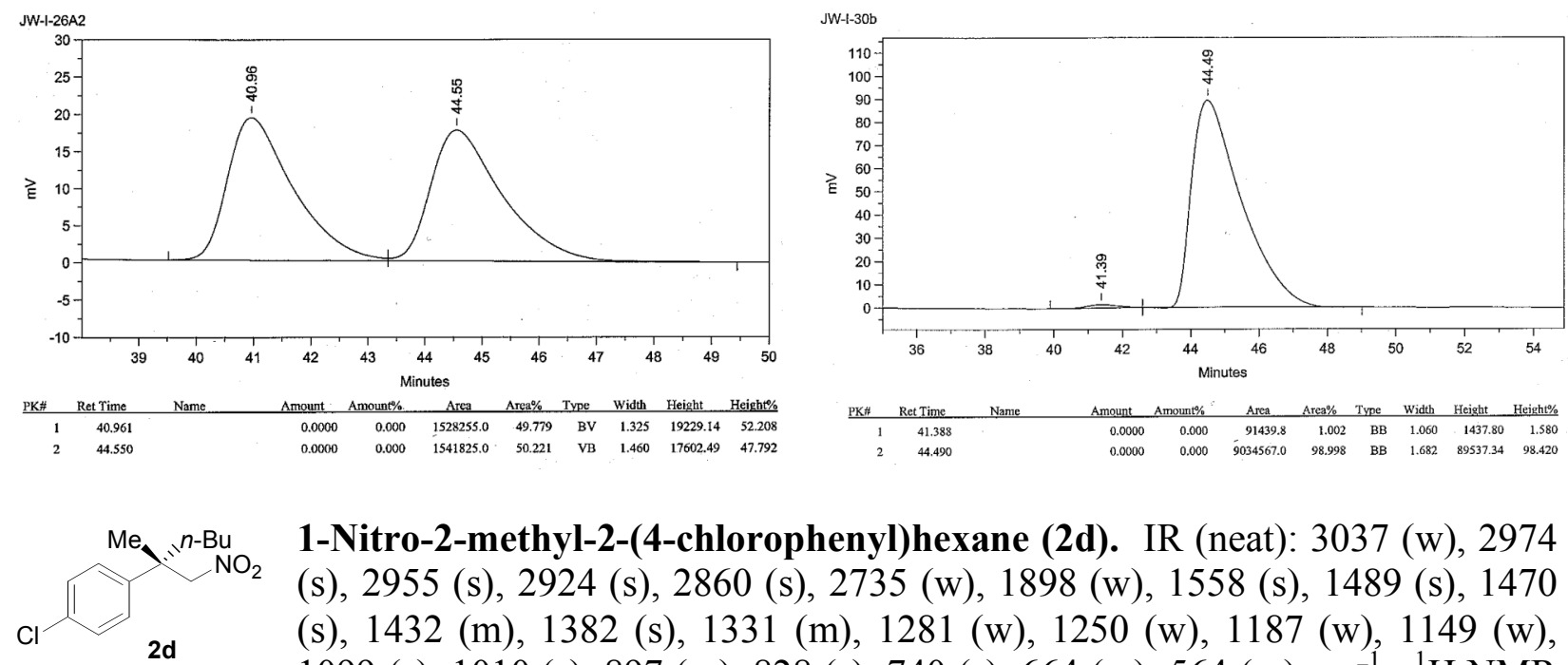

1-Nitro-2-methyl-2-(4-chlorophenyl)hexane (2d). IR (neat): 3037 (w), 2974 (s), 2955 (s), 2924 (s), 2860 (s), 2735 (w), 1898 (w), 1558 (s), 1489 (s), 1470 (s), $1432(\mathrm{~m}), 1382(\mathrm{~s}), 1331(\mathrm{~m}), 1281(\mathrm{w}), 1250(\mathrm{w}), 1187(\mathrm{w}), 1149(\mathrm{w})$, 1099 (s), 1010 (s), 897 (m), 828 (s), 740 (s), $664(\mathrm{~m}), 564(\mathrm{~m}) \mathrm{cm}^{-1}$. ${ }^{1} \mathrm{H}$ NMR $\left(\mathrm{CDCl}_{3}, 400 \mathrm{MHz}\right): \delta$ 7.32-7.29 $(2 \mathrm{H}, \mathrm{m}, \mathrm{ArH}), 7.24-7.21(2 \mathrm{H}, \mathrm{m}, \operatorname{ArH}), 4.56(1 \mathrm{H}, \mathrm{d}, J=$ $\left.10.8 \mathrm{~Hz}, \mathrm{CHHNO}_{2}\right), 4.49\left(1 \mathrm{H}, \mathrm{d}, J=10.8 \mathrm{~Hz}, \mathrm{CHHNO}_{2}\right), 1.82(1 \mathrm{H}, \mathrm{dt}, J=13.0,4.4 \mathrm{~Hz}$, $\left.\mathrm{CHH}\left(\mathrm{CH}_{2}\right)_{2} \mathrm{CH}_{3}\right), 1.64\left(1 \mathrm{H}, \mathrm{dt}, J=13.0,4.4 \mathrm{~Hz}, \mathrm{CHH}\left(\mathrm{CH}_{2}\right)_{2} \mathrm{CH}_{3}\right), 1.51\left(3 \mathrm{H}, \mathrm{s}, \mathrm{CCH}_{3}\right), 1.29-$ $0.90\left(4 \mathrm{H}, \mathrm{m}, \mathrm{CH}_{2}\left(\mathrm{CH}_{2}\right)_{2} \mathrm{CH}_{3}\right), 0.82\left(3 \mathrm{H}, \mathrm{t}, J=7.2 \mathrm{~Hz},\left(\mathrm{CH}_{2}\right)_{3} \mathrm{CH}_{3}\right) .{ }^{13} \mathrm{C} \mathrm{NMR}\left(\mathrm{CDCl}_{3}, 100\right.$ MHz): $\delta 141.1,133.0,128.9,127.7,86.3,42.3,39.7,26.0,23.1,22.5,14.0$. HRMS Calcd for 
$\mathrm{C}_{13} \mathrm{H}_{18} \mathrm{ClNO}_{2}$ : 255.1026. Found: 255.1035. Anal. Calcd for $\mathrm{C}_{13} \mathrm{H}_{18} \mathrm{ClNO}_{2}$ : $\mathrm{C}, 61.05 ; \mathrm{H}, 7.09$; $\mathrm{N}, 5.48$. Found: $\mathrm{C}, 61.28 ; \mathrm{H}, 7.32 ; \mathrm{N}, 5.26 .[\alpha]_{\mathrm{D}}{ }^{20}-37.6^{\circ}\left(c=0.910, \mathrm{CHCl}_{3}\right)$ for a sample that is $96 \%$ ee.

Optical purity is established by chiral HPLC analysis (Chiral Technologies Chiralpak OD, $254 \mathrm{~nm}, 98: 2$ hexanes $/ i-\mathrm{PrOH}, 0.3 \mathrm{~mL} / \mathrm{min}$ ); chromatograms are illustrated below for a $96 \%$ ee sample:
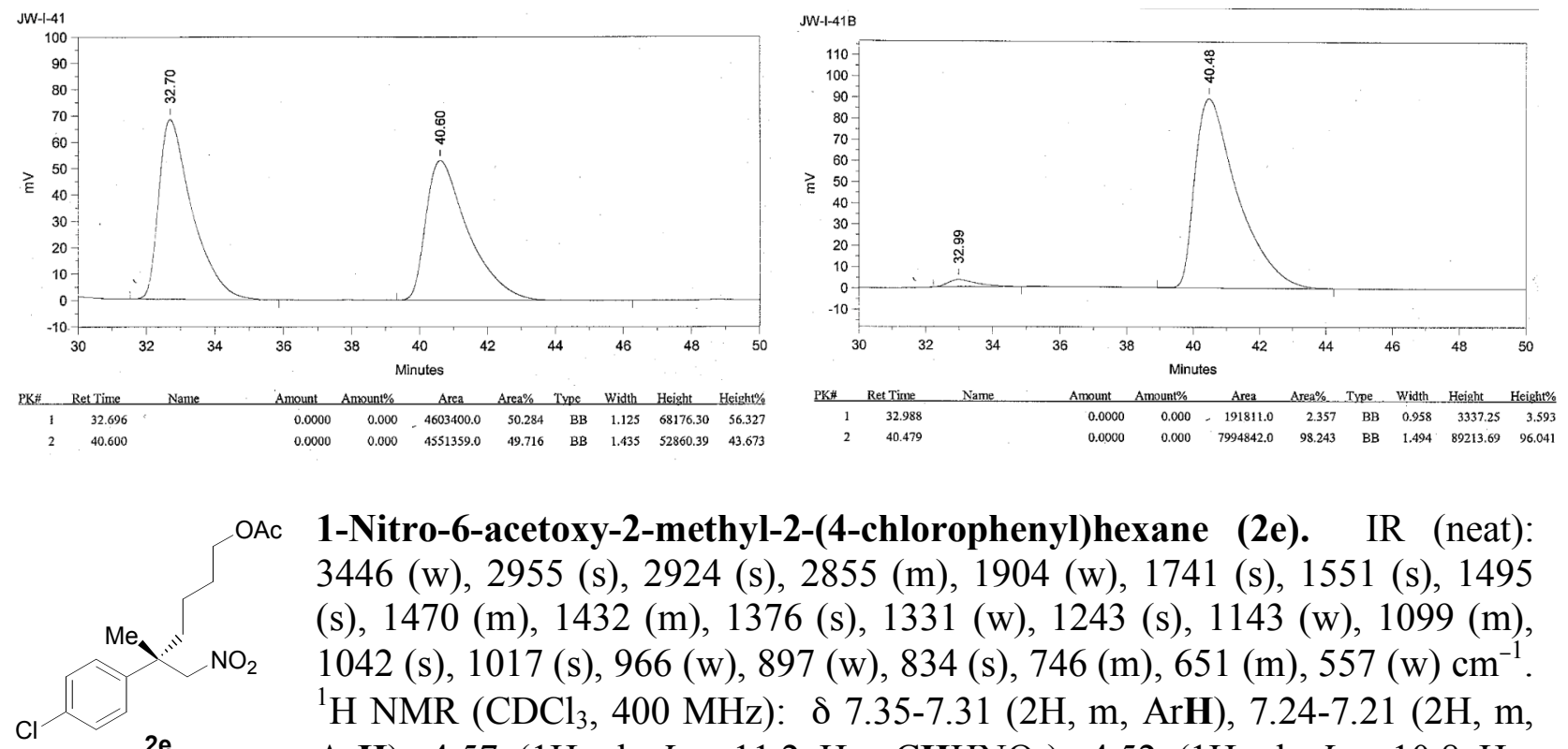

1-Nitro-6-acetoxy-2-methyl-2-(4-chlorophenyl)hexane (2e). IR (neat): $3446(\mathrm{w}), 2955$ (s), $2924(\mathrm{~s}), 2855(\mathrm{~m}), 1904(\mathrm{w}), 1741(\mathrm{~s}), 1551(\mathrm{~s}), 1495$ (s), $1470(\mathrm{~m}), 1432(\mathrm{~m}), 1376(\mathrm{~s}), 1331(\mathrm{w}), 1243(\mathrm{~s}), 1143(\mathrm{w}), 1099(\mathrm{~m})$ $1042(\mathrm{~s}), 1017(\mathrm{~s}), 966(\mathrm{w}), 897(\mathrm{w}), 834(\mathrm{~s}), 746(\mathrm{~m}), 651(\mathrm{~m}), 557(\mathrm{w}) \mathrm{cm}^{-1}$. ${ }^{1} \mathrm{H}$ NMR $\left(\mathrm{CDCl}_{3}, 400 \mathrm{MHz}\right): \delta$ 7.35-7.31 $(2 \mathrm{H}, \mathrm{m}, \mathrm{ArH}), 7.24-7.21(2 \mathrm{H}, \mathrm{m}$, $\operatorname{ArH}), 4.57\left(1 \mathrm{H}, \mathrm{d}, J=11.2 \mathrm{~Hz}, \mathrm{CHHNO}_{2}\right), 4.52(1 \mathrm{H}, \mathrm{d}, J=10.8 \mathrm{~Hz}$, $\left.\mathrm{CHHNO}_{2}\right), 3.98\left(2 \mathrm{H}, \mathrm{t}, J=6.8 \mathrm{~Hz}, \mathrm{CH}_{2} \mathrm{OAc}\right), 2.00\left(3 \mathrm{H}, \mathrm{s}, \mathrm{OCOCH}_{3}\right), 1.88(1 \mathrm{H}, \mathrm{dt}, J=13.0,4.4$ $\left.\mathrm{Hz}, \mathrm{CHH}\left(\mathrm{CH}_{2}\right)_{3} \mathrm{OAc}\right), 1.70\left(1 \mathrm{H}, \mathrm{dt}, J=13.0,4.4 \mathrm{~Hz}, \mathrm{CHH}\left(\mathrm{CH}_{2}\right)_{3} \mathrm{OAc}\right), 1.60-1.01(7 \mathrm{H}, \mathrm{m}$, $\mathrm{CCH}_{3}$ and $\left.\mathrm{CH}_{2}\left(\mathrm{CH}_{2}\right)_{2} \mathrm{CH}_{2} \mathrm{OAc}\right) .{ }^{13} \mathrm{C} \mathrm{NMR}\left(\mathrm{CDCl}_{3}, 100 \mathrm{MHz}\right): \delta 171.2,140.7,133.2,129.0$, 127.7, 86.2, 63.9, 42.3, 39.4, 28.9, 22.5, 21.1, 20.4. HRMS Calcd for $\mathrm{C}_{15} \mathrm{H}_{20} \mathrm{ClNO}_{4}$ : 313.1081 . Found: 313.1084 . $[\alpha]_{\mathrm{D}}{ }^{20}-22.0^{\circ}\left(c=0.907, \mathrm{CHCl}_{3}\right)$ for a sample that is $85 \%$ ee.

Optical purity is established by chiral HPLC analysis (Chiral Technologies Chiralpak OD, $254 \mathrm{~nm}, 99: 1$ hexanes $/ i-\mathrm{PrOH}, 0.3 \mathrm{~mL} / \mathrm{min}$ ); chromatograms are illustrated below for a $85 \%$ ee sample:
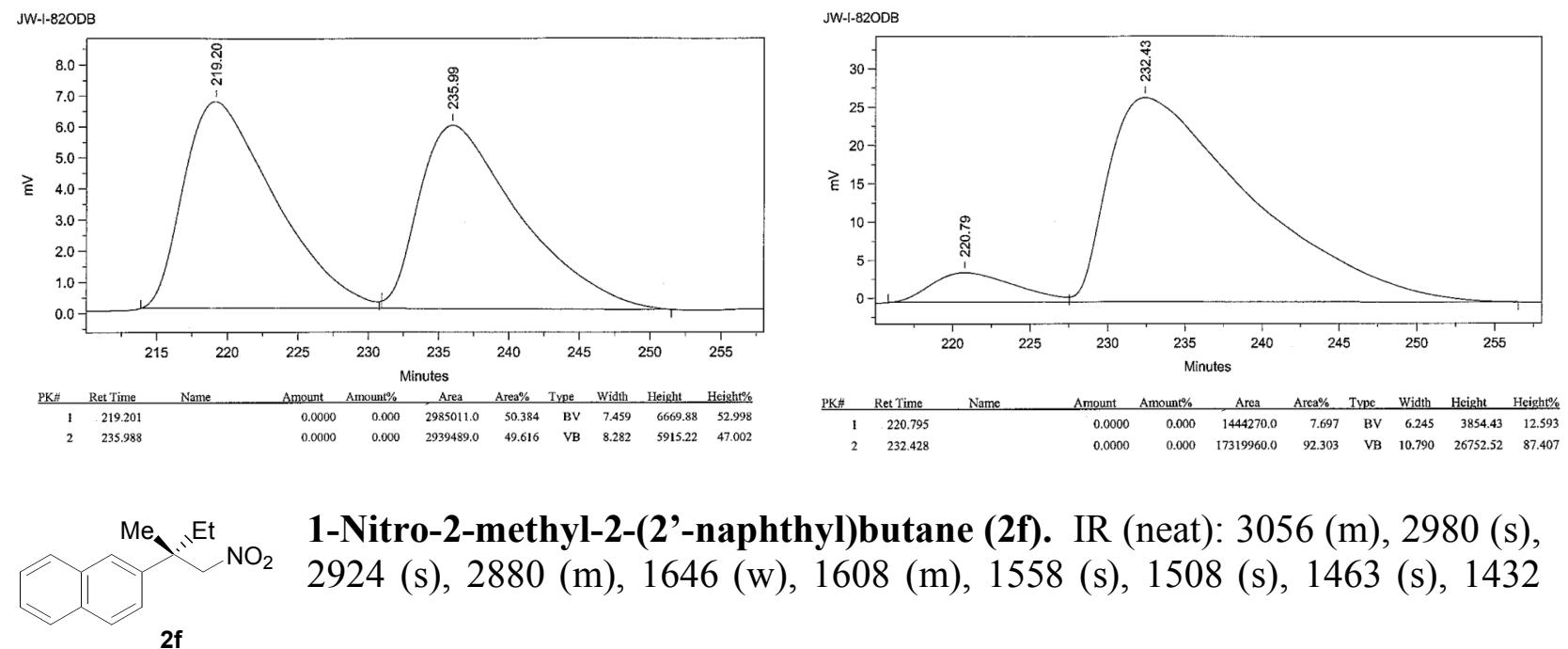
(m), $1388(\mathrm{~s}), 1331(\mathrm{~m}), 1268(\mathrm{~m}), 1143(\mathrm{w}), 954(\mathrm{w}), 821(\mathrm{~s}), 790(\mathrm{~m}), 765(\mathrm{~m}), 740(\mathrm{~s}), 721(\mathrm{~s})$, $664(\mathrm{~m}), 551(\mathrm{~m}) \mathrm{cm}^{-1} .{ }^{1} \mathrm{H} \mathrm{NMR}\left(\mathrm{CDCl}_{3}, 400 \mathrm{MHz}\right): \delta 7.87-7.82(3 \mathrm{H}, \mathrm{m}, \mathrm{ArH}), 7.71(1 \mathrm{H}, \mathrm{d}, J$ $=2.0 \mathrm{~Hz}, \operatorname{ArH}), 7.52-7.45(3 \mathrm{H}, \mathrm{m}, \operatorname{ArH}), 4.71\left(1 \mathrm{H}, \mathrm{d}, J=10.8 \mathrm{~Hz}, \mathrm{CHHNO}_{2}\right), 4.66(1 \mathrm{H}, \mathrm{d}, J=$ $\left.10.8 \mathrm{~Hz}, \mathrm{CHHNO}_{2}\right), 2.07\left(1 \mathrm{H}, \mathrm{dq}, J=14.4,7.2 \mathrm{~Hz}, \mathrm{CHHCH}_{3}\right), 1.83(1 \mathrm{H}, \mathrm{dq}, J=14.4,7.2 \mathrm{~Hz}$, $\left.\mathrm{CHHCH}_{3}\right), 1.65\left(3 \mathrm{H}, \mathrm{s}, \mathrm{CCH}_{3}\right), 0.76\left(3 \mathrm{H}, \mathrm{t}, J=7.2 \mathrm{~Hz}, \mathrm{CH}_{2} \mathrm{CH}_{3}\right) .{ }^{13} \mathrm{C} \mathrm{NMR}\left(\mathrm{CDCl}_{3}, 100 \mathrm{MHz}\right)$ : $\delta$ 139.5, 133.4, 132.4, 128.5, 128.2, 127.6, 126.4, 126.3, 125.7, 124.1, 86.2, 43.1, 32.4, 22.1, 8.4. HRMS Calcd for $\mathrm{C}_{15} \mathrm{H}_{17} \mathrm{NO}_{2}$ : 243.1259. Found: 243.1259. Anal. Calcd for $\mathrm{C}_{15} \mathrm{H}_{17} \mathrm{NO}_{2}$ : C, 74.05; H, 7.04; N, 5.76. Found: C, 74.31; H, 6.82; N, 5.49. $[\alpha]_{\mathrm{D}}{ }^{20}-53.7^{\circ}\left(c=0.940, \mathrm{CHCl}_{3}\right)$ for a sample that is $95 \%$ ee.

Optical purity is established by chiral HPLC analysis (Chiral Technologies Chiralpak OD, $254 \mathrm{~nm}, 98: 2$ hexanes $/ i-\mathrm{PrOH}, 1.0 \mathrm{~mL} / \mathrm{min}$ ); chromatograms are illustrated below for a $95 \%$ ee sample:
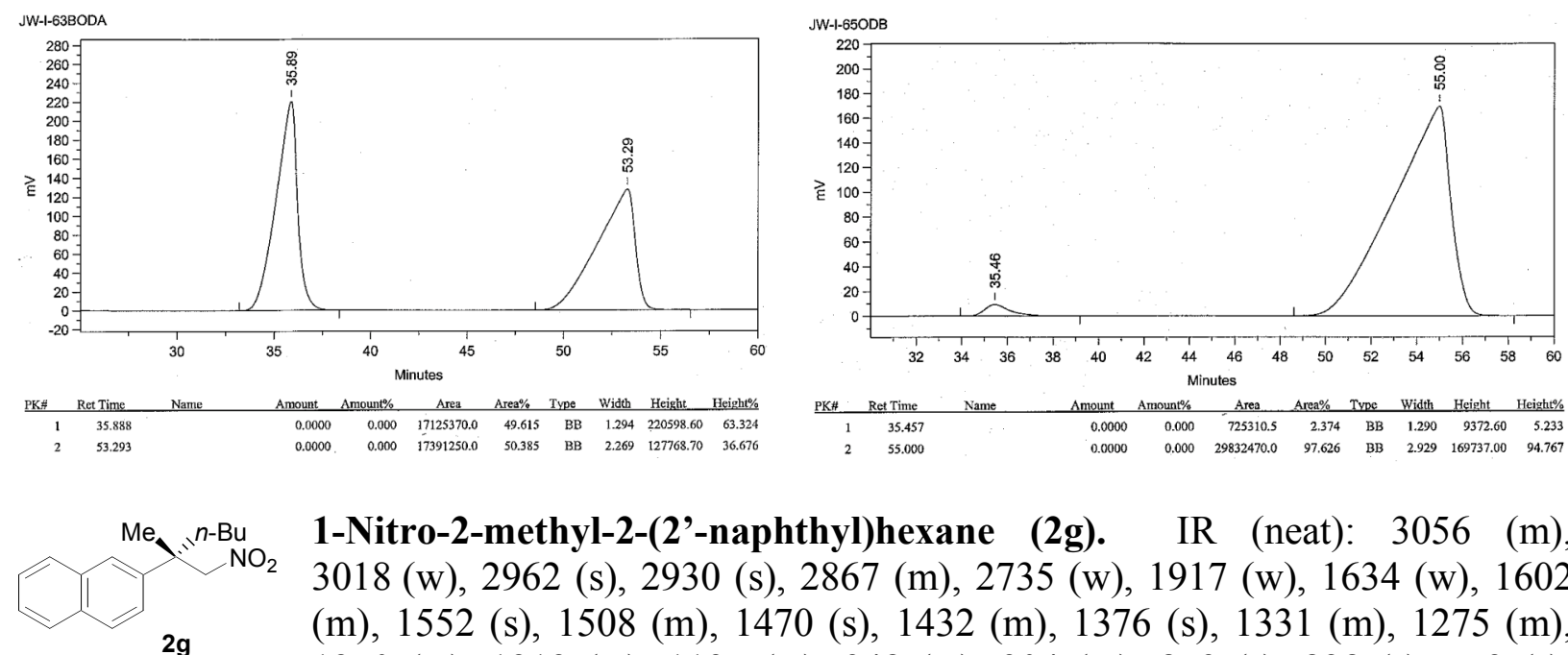

1-Nitro-2-methyl-2-(2'-naphthyl)hexane (2g). $\quad$ IR (neat): 3056 (m), 3018 (w), 2962 (s), 2930 (s), 2867 (m), 2735 (w), 1917 (w), 1634 (w), 1602 (m), 1552 (s), 1508 (m), 1470 (s), 1432 (m), 1376 (s), 1331 (m), 1275 (m), $1250(\mathrm{w}), 1212(\mathrm{w}), 1137(\mathrm{w}), 948(\mathrm{w}), 904(\mathrm{~m}), 859$ (s), 828 (s), 752 (s), $671(\mathrm{~m}), 476(\mathrm{~m}) \mathrm{cm}^{-1} .{ }^{1} \mathrm{H}$ NMR $\left(\mathrm{CDCl}_{3}, 400 \mathrm{MHz}\right): \delta 7.87-7.82(3 \mathrm{H}, \mathrm{m}, \mathrm{ArH}), 7.71(1 \mathrm{H}, \mathrm{d}, J$ $=1.6 \mathrm{~Hz}, \operatorname{ArH}), 7.52-7.45(3 \mathrm{H}, \mathrm{m}, \operatorname{ArH}), 4.70\left(1 \mathrm{H}, \mathrm{d}, J=10.8 \mathrm{~Hz}, \mathrm{CHHNO}_{2}\right), 4.65(1 \mathrm{H}, \mathrm{d}, J=$ $\left.10.8 \mathrm{~Hz}, \mathrm{CHHNO}_{2}\right), 2.00\left(1 \mathrm{H}, \mathrm{dt}, J=13.0,4.0 \mathrm{~Hz}, \mathrm{CHH}\left(\mathrm{CH}_{2}\right)_{2} \mathrm{CH}_{3}\right), 1.76(1 \mathrm{H}, \mathrm{dt}, J=13.0,4.4$ $\left.\mathrm{Hz}, \mathrm{CHH}\left(\mathrm{CH}_{2}\right)_{2} \mathrm{CH}_{3}\right), 1.67\left(3 \mathrm{H}, \mathrm{s}, \mathrm{CCH}_{3}\right), 1.31-0.92\left(4 \mathrm{H}, \mathrm{m}, \mathrm{CH}_{2}\left(\mathrm{CH}_{2}\right)_{2} \mathrm{CH}_{3}\right), 0.82(3 \mathrm{H}, \mathrm{t}, J=$ $\left.7.2 \mathrm{~Hz},\left(\mathrm{CH}_{2}\right)_{3} \mathrm{CH}_{3}\right) .{ }^{13} \mathrm{C} \mathrm{NMR}\left(\mathrm{CDCl}_{3}, 100 \mathrm{MHz}\right): \delta \mathrm{139.9}, 133.4,132.3,128.5,128.2,127.6$, 126.4, 126.3, 125.5, 124.0, 86.4, 42.8, 39.5, 26.2, 23.2, 22.6, 14.0. HRMS Calcd for $\mathrm{C}_{17} \mathrm{H}_{21} \mathrm{NO}_{2}$ : 271.1572. Found: 271.1576. Anal. Calcd for $\mathrm{C}_{17} \mathrm{H}_{21} \mathrm{NO}_{2}$ : C, 75.25; H, 7.80; $\mathrm{N}, 5.16$. Found: $\mathrm{C}$, $74.98 ; \mathrm{H}, 8.05 ; \mathrm{N}, 4.99$. $[\alpha]_{\mathrm{D}}{ }^{20}-51.2^{\circ}\left(c=0.57, \mathrm{CHCl}_{3}\right)$ for a sample that is $85 \%$ ee.

The optical purity is established by chiral HPLC analysis (Chiral Technologies Chiralpak OD, $254 \mathrm{~nm}, 97: 3$ hexanes $/ i-\mathrm{PrOH}, 1.0 \mathrm{~mL} / \mathrm{min}$ ); chromatograms are illustrated below for an $85 \%$ ee sample: 

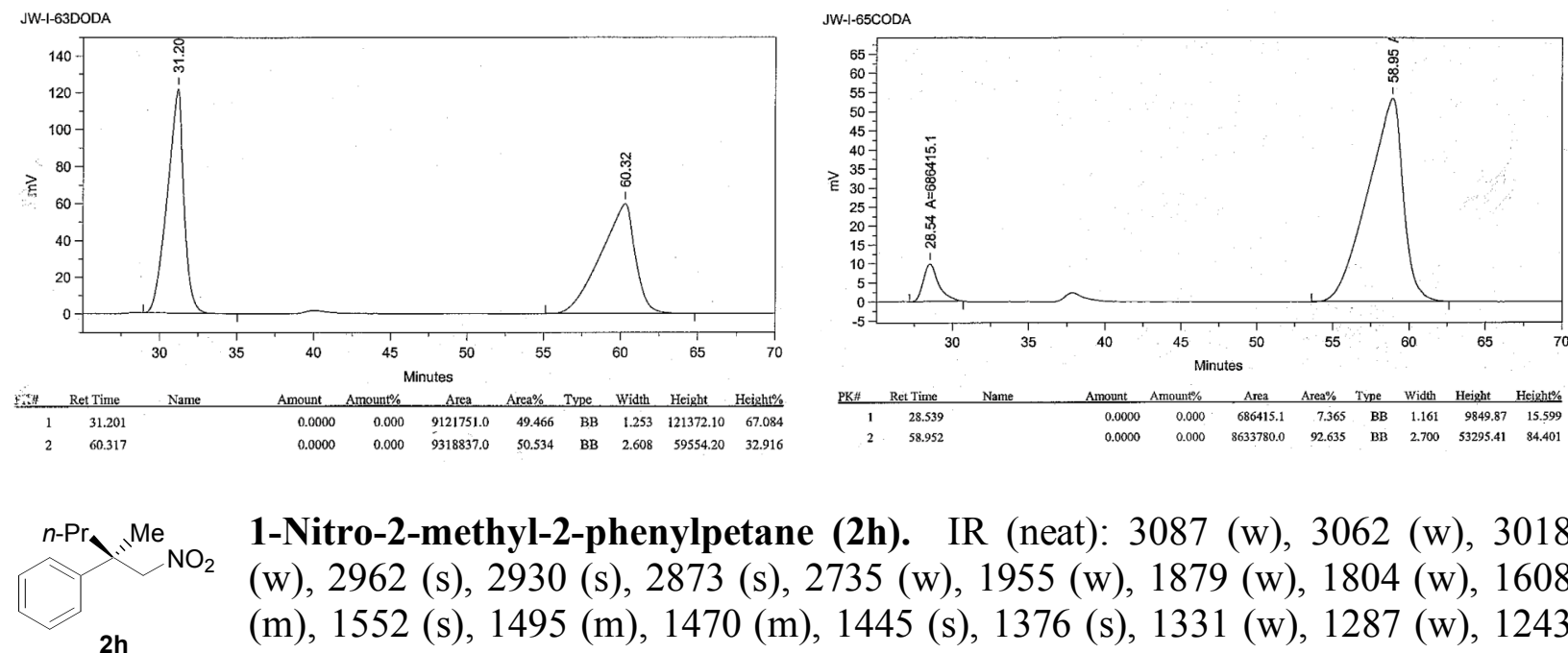

1-Nitro-2-methyl-2-phenylpetane (2h). IR (neat): 3087 (w), 3062 (w), 3018 (w), 2962 (s), 2930 (s), 2873 (s), 2735 (w), 1955 (w), 1879 (w), 1804 (w), 1608 (m), 1552 (s), 1495 (m), $1470(\mathrm{~m}), 1445$ (s), 1376 (s), $1331(\mathrm{w}), 1287(\mathrm{w}), 1243$ (w), $1199(\mathrm{w}), 1149(\mathrm{w}), 1036(\mathrm{~m}), 841(\mathrm{w}), 771(\mathrm{~m}), 746(\mathrm{~m}), 702(\mathrm{~s}), 652(\mathrm{~m})$, $576(\mathrm{~m}) \mathrm{cm}^{-1}$. ${ }^{1} \mathrm{H} \mathrm{NMR}\left(\mathrm{CDCl}_{3}, 400 \mathrm{MHz}\right): \delta 7.38-7.54(5 \mathrm{H}, \mathrm{m}, \mathrm{ArH}), 4.59(1 \mathrm{H}, \mathrm{d}, J=10.8$ $\left.\mathrm{Hz}, \mathrm{CHHNO}_{2}\right), 4.55\left(1 \mathrm{H}, \mathrm{d}, J=10.8 \mathrm{~Hz}, \mathrm{CHHNO}_{2}\right), 1.87(1 \mathrm{H}, \mathrm{dt}, J=13.0,4.4 \mathrm{~Hz}$, $\left.\mathrm{CHHCH}_{2} \mathrm{CH}_{3}\right), 1.68\left(1 \mathrm{H}, \mathrm{dt}, J=12.8,4.4 \mathrm{~Hz} \mathrm{CHHCH}_{2} \mathrm{CH}_{3}\right), 1.55\left(3 \mathrm{H}, \mathrm{s}, \mathrm{CCH}_{3}\right), 1.26-1.16$ $\left(1 \mathrm{H}, \mathrm{m}, \mathrm{CH}_{2} \mathrm{CHHCH}_{3}\right), 1.10-0.97\left(1 \mathrm{H}, \mathrm{m}, \mathrm{CH}_{2} \mathrm{CHHCH}_{3}\right), 0.87\left(3 \mathrm{H}, \mathrm{t}, J=7.2 \mathrm{~Hz},\left(\mathrm{CH}_{2}\right)_{2} \mathrm{CH}_{3}\right)$. ${ }^{13} \mathrm{C} \mathrm{NMR}\left(\mathrm{CDCl}_{3}, 100 \mathrm{MHz}\right): \delta 142.6,128.8,127.1,126.2,86.5,42.6,42.2,22.6,17.3,14.6$. HRMS Calcd for $\mathrm{C}_{12} \mathrm{H}_{17} \mathrm{NO}_{2}$ : 207.1259. Found: 207.1259. Anal. Calcd for $\mathrm{C}_{12} \mathrm{H}_{17} \mathrm{NO}_{2}$ : $\mathrm{C}$, 69.54; H, 8.27; N, 6.76. Found: C, 69.48; H, 8.47; N, 6.48. $[\alpha]_{\mathrm{D}}{ }^{20}-41.9^{\circ}\left(c=0.45, \mathrm{CHCl}_{3}\right)$ for a sample that is $85 \%$ ee.

Optical purity is established by chiral GLC analysis (Chiraldex GTA, $128{ }^{\circ} \mathrm{C}, 20 \mathrm{psi}$ ); chromatograms are illustrated below for a $85 \%$ ee sample:
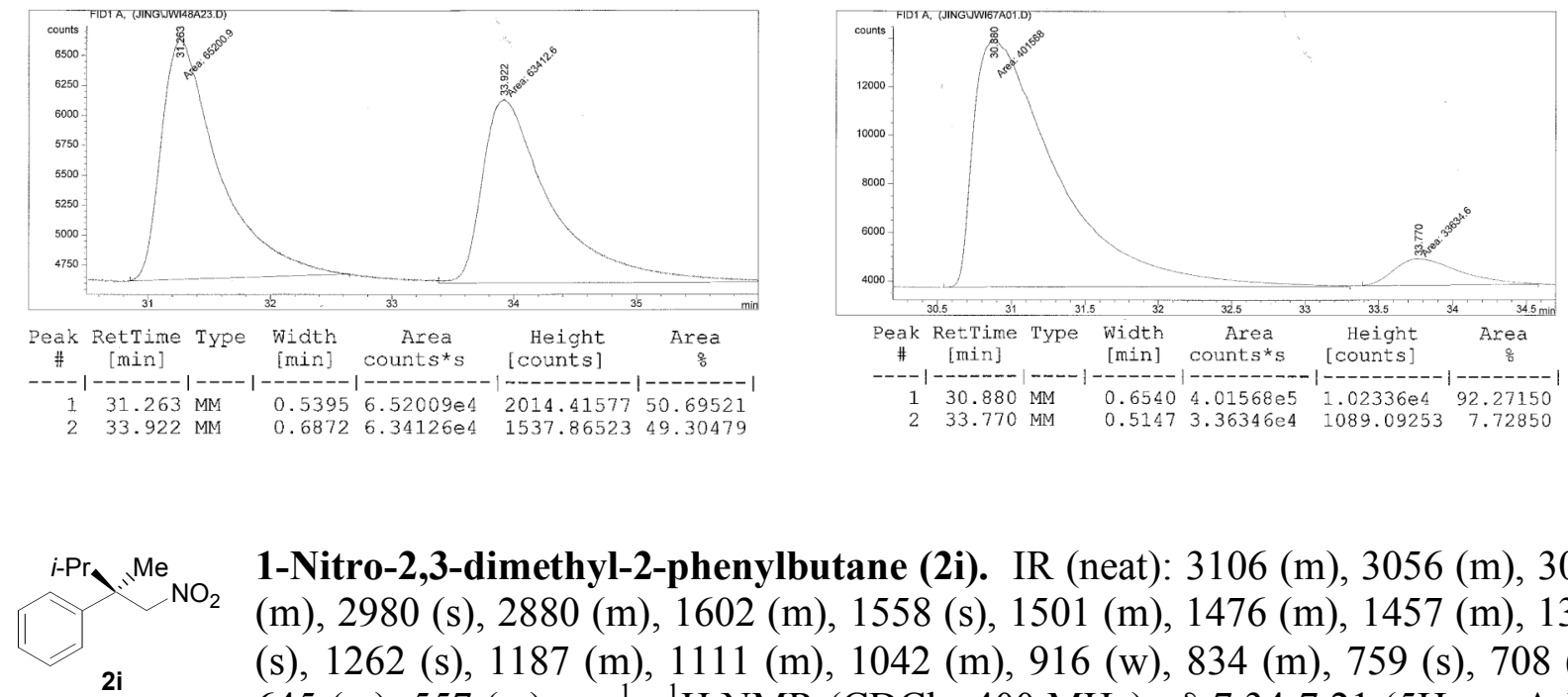

1-Nitro-2,3-dimethyl-2-phenylbutane (2i). IR (neat): 3106 (m), 3056 (m), 3037 (m), $2980(\mathrm{~s}), 2880(\mathrm{~m}), 1602(\mathrm{~m}), 1558(\mathrm{~s}), 1501(\mathrm{~m}), 1476(\mathrm{~m}), 1457(\mathrm{~m}), 1382$ (s), 1262 (s), $1187(\mathrm{~m}), 1111(\mathrm{~m}), 1042(\mathrm{~m}), 916(\mathrm{w}), 834(\mathrm{~m}), 759$ (s), $708(\mathrm{~s})$, $645(\mathrm{~m}), 557(\mathrm{~m}) \mathrm{cm}^{-1}$. ${ }^{1} \mathrm{H} \mathrm{NMR}\left(\mathrm{CDCl}_{3}, 400 \mathrm{MHz}\right)$ : $\delta$ 7.34-7.21 (5H, m, ArH), $4.80\left(1 \mathrm{H}, \mathrm{d}, J=11.2 \mathrm{~Hz}, \mathrm{CHHNO}_{2}\right), 4.61\left(1 \mathrm{H}, \mathrm{d}, J=11.2 \mathrm{~Hz}, \mathrm{CHHNO}_{2}\right), 2.13(1 \mathrm{H}, \mathrm{qq}, J=$ $\left.6.8 \mathrm{~Hz}, \mathrm{CH}\left(\mathrm{CH}_{3}\right)_{2}\right), 1.50\left(3 \mathrm{H}, \mathrm{s}, \operatorname{ArCCH}_{3}\right), 0.94\left(3 \mathrm{H}, \mathrm{d}, J=6.8 \mathrm{~Hz}, \mathrm{CH}_{3} \mathrm{CHCH}_{3}\right), 0.67(3 \mathrm{H}, \mathrm{d}, J$ $\left.=6.8 \mathrm{~Hz}, \mathrm{CH}_{3} \mathrm{CHCH}_{3}\right) .{ }^{13} \mathrm{C} \mathrm{NMR}\left(\mathrm{CDCl}_{3}, 100 \mathrm{MHz}\right): \delta 142.2,128.4,127.0,126.6,85.2,45.7$, 36.8, 17.9, 17.6, 17.0. HRMS Calcd. for $\mathrm{C}_{12} \mathrm{H}_{17} \mathrm{NO}_{2}$ : 207.1259. Found: 207.1259. $[\alpha]_{\mathrm{D}}{ }^{20}$ $-53.1^{\circ}\left(c=3.70, \mathrm{CHCl}_{3}\right)$ for a sample that is $73 \%$ ee. 
Optical purity is established by chiral GLC analysis (Chiraldex GTA, $140{ }^{\circ} \mathrm{C}, 15 \mathrm{psi}$ ); chromatograms are illustrated below for a $73 \%$ ee sample:
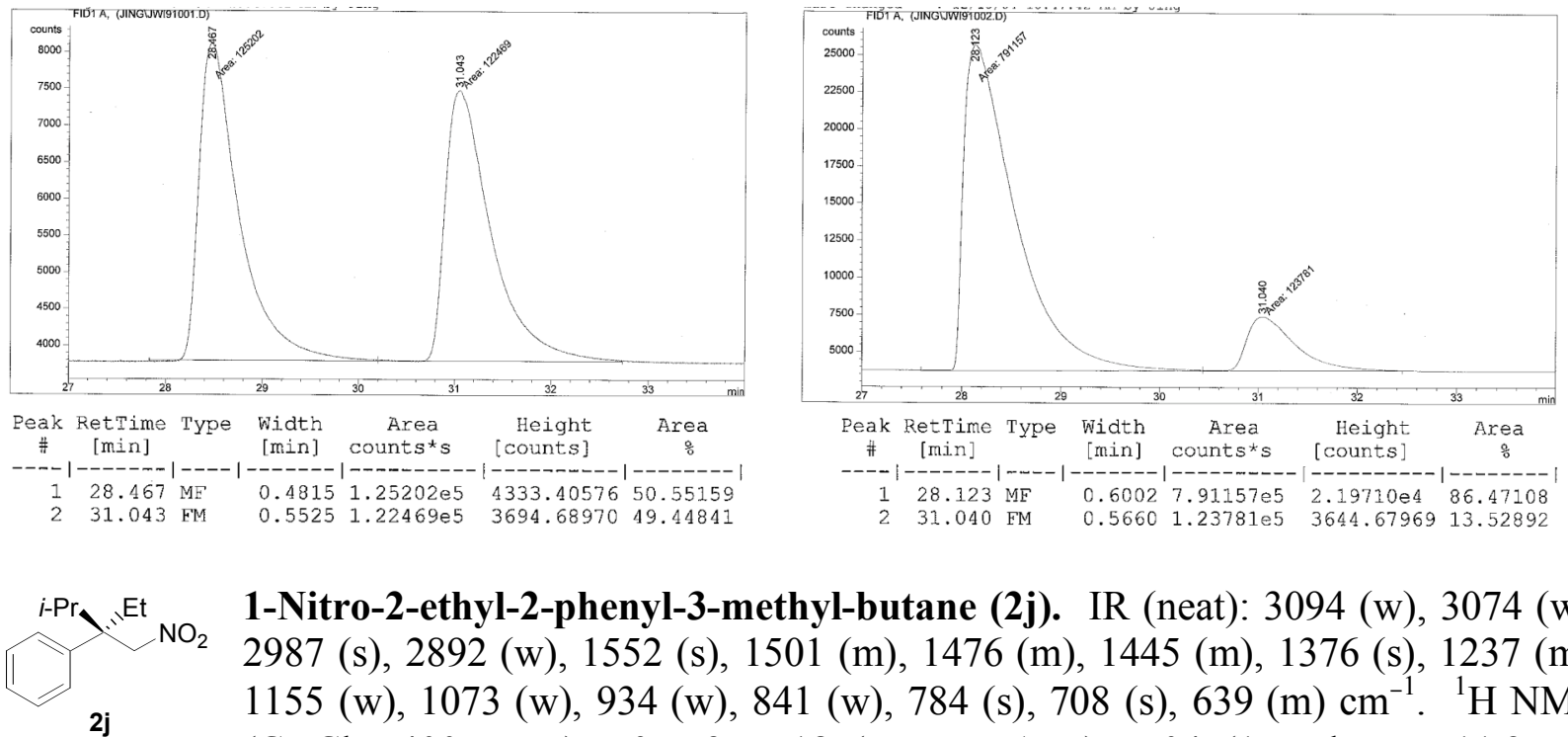

1-Nitro-2-ethyl-2-phenyl-3-methyl-butane (2j). IR (neat): 3094 (w), 3074 (w), 2987 (s), 2892 (w), 1552 (s), 1501 (m), 1476 (m), 1445 (m), 1376 (s), 1237 (m), 1155 (w), 1073 (w), 934 (w), 841 (w), 784 (s), 708 (s), 639 (m) cm ${ }^{-1}$. ${ }^{1} \mathrm{H}$ NMR $\left(\mathrm{CDCl}_{3}, 400 \mathrm{MHz}\right): \delta$ 7.37-7.18 $(5 \mathrm{H}, \mathrm{m}, \mathrm{ArH}), 5.04(1 \mathrm{H}, \mathrm{d}, J=11.2 \mathrm{~Hz}$, $\left.\mathrm{CHHNO}_{2}\right), 4.84\left(1 \mathrm{H}, \mathrm{d}, J=11.2 \mathrm{~Hz}, \mathrm{CHHNO}_{2}\right), 2.23\left(1 \mathrm{H}, \mathrm{dq}, J=14.8,7.6 \mathrm{~Hz}, \mathrm{CCHHCH}_{3}\right)$, $2.08\left(1 \mathrm{H}, \mathrm{m}, \mathrm{CH}\left(\mathrm{CH}_{3}\right)_{2}\right), 1.87\left(1 \mathrm{H}, \mathrm{dq}, J=14.8,7.6 \mathrm{~Hz}, \mathrm{CCHHCH}_{3}\right), 0.92(3 \mathrm{H}, \mathrm{t}, J=7.2 \mathrm{~Hz}$, $\left.\mathrm{CH}_{2} \mathrm{CH}_{3}\right), 0.87\left(3 \mathrm{H}, \mathrm{d}, J=6.8 \mathrm{~Hz}, \mathrm{CH}_{3} \mathrm{CHCH}_{3}\right), 0.79\left(3 \mathrm{H}, \mathrm{d}, J=6.8 \mathrm{~Hz}, \mathrm{CH}_{3} \mathrm{CHCH}_{3}\right) .{ }^{13} \mathrm{C}$ $\mathrm{NMR}\left(\mathrm{CDCl}_{3}, 100 \mathrm{MHz}\right): \delta 128.2,127.5,126.8,78.6,49.1,34.1,26.2,17.9,17.6,8.7 . \mathrm{HRMS}$ Calcd for $\mathrm{C}_{13} \mathrm{H}_{19} \mathrm{NO}_{2}$ : 221.1416. Found: 221.1416. Anal. Calcd for $\mathrm{C}_{13} \mathrm{H}_{19} \mathrm{NO}_{2}$ : C, 70.56; $\mathrm{H}$, 8.65; N, 6.33. Found: C, 70.27; H, 8.33; N, 6.05. $[\alpha]_{\mathrm{D}}{ }^{20}-4.4^{\circ}\left(c=4.50, \mathrm{CHCl}_{3}\right)$ for a sample that is $93 \%$ ee.

Optical purity is established by chiral GLC analysis $\left(\beta-d e x, 130{ }^{\circ} \mathrm{C}, 20 \mathrm{psi}\right)$; chromatograms are illustrated below for a $93 \%$ ee sample:
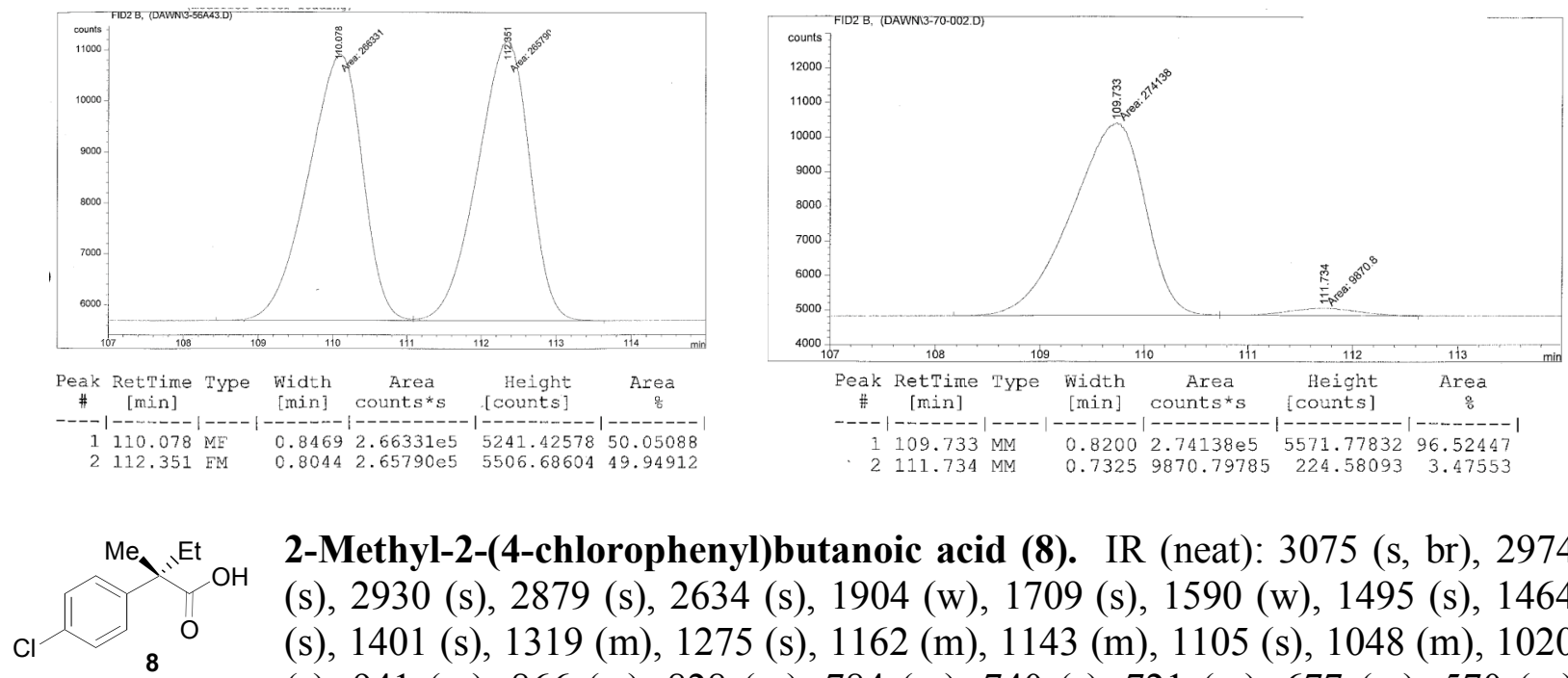

2-Methyl-2-(4-chlorophenyl)butanoic acid (8). IR (neat): 3075 (s, br), 2974 (s), 2930 (s), 2879 (s), 2634 (s), 1904 (w), 1709 (s), 1590 (w), 1495 (s), 1464 (s), $1401(\mathrm{~s}), 1319(\mathrm{~m}), 1275(\mathrm{~s}), 1162(\mathrm{~m}), 1143(\mathrm{~m}), 1105(\mathrm{~s}), 1048(\mathrm{~m}), 1020$ (s), $941(\mathrm{~m}), 866(\mathrm{w}), 828(\mathrm{~m}), 784(\mathrm{~m}), 740(\mathrm{~s}), 721(\mathrm{~m}), 677(\mathrm{~m}), 570(\mathrm{~m})$ $\mathrm{cm}^{-1} .{ }^{1} \mathrm{H}$ NMR $\left(\mathrm{CDCl}_{3}, 400 \mathrm{MHz}\right): \delta 7.31(4 \mathrm{H}, \mathrm{s}, \mathrm{ArH}), 2.07(1 \mathrm{H}, \mathrm{dq}, J=14.4,7.2 \mathrm{~Hz}$, $\left.\mathrm{CHHCH}_{3}\right), 1.96\left(1 \mathrm{H}, \mathrm{dq}, J=14.4,7.2 \mathrm{~Hz}, \mathrm{CHHCH}_{3}\right), 1.54\left(3 \mathrm{H}, \mathrm{s}, \mathrm{CCH}_{3}\right), 0.83(3 \mathrm{H}, \mathrm{t}, J=7.2$ 
$\left.\mathrm{Hz}, \mathrm{CH}_{2} \mathrm{CH}_{3}\right) .{ }^{13} \mathrm{C} \mathrm{NMR}\left(\mathrm{CDCl}_{3}, 100 \mathrm{MHz}\right): \delta 182.1,141.4,133.0,128.7,128.0,50.2,31.8$, 21.2, 9.1. HRMS Calcd for $\mathrm{C}_{11} \mathrm{H}_{13} \mathrm{ClO}_{2}$ : 212.0604. Found: 212.0604. Anal. Calcd for $\mathrm{C}_{11} \mathrm{H}_{13} \mathrm{ClO}_{2}$ : C, 62.12; $\mathrm{H}, 6.16$. Found: $\mathrm{C}, 62.12 ; \mathrm{H}, 6.16 .[\alpha]_{\mathrm{D}}{ }^{20}+10.0^{\circ}\left(c=0.88, \mathrm{CHCl}_{3}\right)$ for a sample that is $98 \%$ ee.

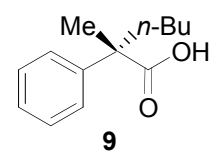

2-Methyl-2-phenylhexanoic acid (9). IR (neat): 3068 (br), 2962 (s), 2923 (s), 2873 (s), 2634 (w), 1948 (w), 1703 (s), 1602 (w), 1552 (w), 1495 (w), 1470 (m), $1451(\mathrm{~m}), 1407$ (m), $1388(\mathrm{~m}), 1281(\mathrm{~s}), 1224(\mathrm{w}), 1162(\mathrm{w}), 1143(\mathrm{w}), 1029(\mathrm{w})$, $941(\mathrm{~m}), 853$ (w), $759(\mathrm{w}), 728(\mathrm{~m}), 702(\mathrm{~s}), 664(\mathrm{w}), 633(\mathrm{w}) \mathrm{cm}^{-1} \cdot{ }^{1} \mathrm{H}$ NMR $\left(\mathrm{CDCl}_{3}, 400 \mathrm{MHz}\right): \delta$ 7.39-7.31 (4H, m, ArH), 7.27-7.23 (1H, m, ArH), $2.04(1 \mathrm{H}, \mathrm{dt}, J=12.6$, $\left.5.2 \mathrm{~Hz}, \mathrm{CHH}\left(\mathrm{CH}_{2}\right)_{2} \mathrm{CH}_{3}\right), 1.93\left(1 \mathrm{H}, \mathrm{dt}, J=12.4,5.2 \mathrm{~Hz} \mathrm{CHH}\left(\mathrm{CH}_{2}\right)_{2} \mathrm{CH}_{3}\right), 1.57\left(3 \mathrm{H}, \mathrm{s}, \mathrm{CCH}_{3}\right)$, 1.35-1.16 (4H, m, $\left.\mathrm{CH}_{2}\left(\mathrm{CH}_{2}\right)_{2} \mathrm{CH}_{3}\right), 0.88\left(3 \mathrm{H}, \mathrm{t}, J=7.2 \mathrm{~Hz},\left(\mathrm{CH}_{2}\right)_{3} \mathrm{CH}_{3}\right) .{ }^{13} \mathrm{C} \mathrm{NMR}\left(\mathrm{CDCl}_{3}, 100\right.$ $\mathrm{MHz}): \delta 181.7,143.3,128.5,127.0,126.3,50.1,38.9,27.0,23.3,22.6,14.1$. HRMS Calcd for $\mathrm{C}_{13} \mathrm{H}_{18} \mathrm{O}_{2}$ : 206.1307. Found: 206.1310. $[\alpha]_{\mathrm{D}}{ }^{20}+8.93^{\circ}\left(c=0.27, \mathrm{CHCl}_{3}\right)$ for a sample that is $93 \%$ ee.

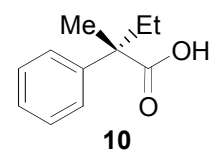

(S)-2-Methyl-2-phenylbutanoic acid (10). IR (neat): 3081 (br), 3056 (s), 3024 (s), 2980 (s), 2936 (s), 2917 (s), 2634 (w), 2663 (w), 1961 (w), 1697 (s), 1602 (w), 1495 (m), 1451 (m), 1401 (m), 1376 (m), 1269 (s), 1162 (m), 1080 (w), 1029 (w), $929(\mathrm{~m}), 803(\mathrm{w}), 771(\mathrm{~m}), 734(\mathrm{~m}), 702(\mathrm{~s}), 633(\mathrm{~m}) \mathrm{cm}^{-1} .{ }^{1} \mathrm{H} \mathrm{NMR}\left(\mathrm{CDCl}_{3}, 400\right.$ $\mathrm{MHz}): \delta$ 7.40-7.32 (4H, m, ArH), 7.28-7.24 (1H, m, ArH), 2.14-1.97 (2H, m, $\left.\mathrm{CH}_{2} \mathrm{CH}_{3}\right), 1.57$ $\left(3 \mathrm{H}, \mathrm{s}, \mathrm{CCH}_{3}\right), 0.86\left(3 \mathrm{H}, \mathrm{t}, J=7.4 \mathrm{~Hz}, \mathrm{CH}_{2} \mathrm{CH}_{3}\right) .{ }^{13} \mathrm{C} \mathrm{NMR}\left(\mathrm{CDCl}_{3}, 100 \mathrm{MHz}\right): \delta 182.7,143.0$, 128.5, 127.0, 126.4, 50.5, 31.8, 21.9, 9.2. HRMS Calcd. for $\mathrm{C}_{11} \mathrm{H}_{14} \mathrm{O}_{2}$ : 178.0994. Found: 178.0995. Anal. Calcd. for $\mathrm{C}_{11} \mathrm{H}_{14} \mathrm{O}_{2}: \mathrm{C}, 74.13 ; \mathrm{H}, 7.92$. Found: $\mathrm{C}, 73.99 ; \mathrm{H}, 7.89 .[\alpha]_{\mathrm{D}}{ }^{20}$ $+23.5^{\circ}\left(c=0.51, \mathrm{C}_{6} \mathrm{H}_{6}\right)$ for a sample that is $94 \%$ ee. Literature value: $[\alpha]_{\mathrm{D}}{ }^{23}+30.2^{\circ}(c=4.5$, $\mathrm{C}_{6} \mathrm{H}_{6}$ ) for optically pure $(S)$ product. $^{6}$

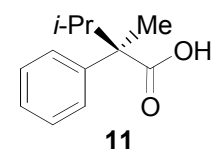

2,3-Dimethyl-2-phenylbutanoic acid (11). IR (neat): 3062 (w), 2968 (s, br), 2924 (s), $2873(\mathrm{~m}), 2848(\mathrm{~m}), 2641(\mathrm{w}), 2521(\mathrm{w}), 1697(\mathrm{~s}), 1602(\mathrm{w}), 1552(\mathrm{~m}), 1495$ (w), $1464(\mathrm{~m}), 1394(\mathrm{~m}), 1376$ (m), 1275 (s), $1130(\mathrm{~m}), 1105$ (m), $929(\mathrm{~m}), 866$ (w), $765(\mathrm{w}), 727(\mathrm{~m}), 696(\mathrm{~s}), 652(\mathrm{~m}) \mathrm{cm}^{-1}$. ${ }^{1} \mathrm{H}$ NMR $\left(\mathrm{CDCl}_{3}, 400 \mathrm{MHz}\right): \delta$ 7.48-7.45 (2H, m, ArH), 7.35-7.30 (2H, m, ArH), 7.25-7.22 (1H, m, ArH), 2.70 (1H, qq, $J=6.4$ $\left.\mathrm{Hz}, \mathrm{CH}\left(\mathrm{CH}_{3}\right)_{2}\right), 1.49\left(3 \mathrm{H}, \mathrm{s}, \mathrm{CCH}_{3}\right), 1.01\left(3 \mathrm{H}, \mathrm{d}, J=6.4 \mathrm{~Hz}, \mathrm{CH}_{3} \mathrm{CHCH}_{3}\right), 0.62(3 \mathrm{H}, \mathrm{d}, J=$ $\left.6.8 \mathrm{~Hz}, \mathrm{CH}_{3} \mathrm{CHCH}_{3}\right) .{ }^{13} \mathrm{C} \mathrm{NMR}\left(\mathrm{CDCl}_{3}, 100 \mathrm{MHz}\right): \delta 182.0,142.2,128.4,127.1,126.8,53.7$, 34.7, 19.1, 17.2, 14.9. HRMS Calcd for $\mathrm{C}_{12} \mathrm{H}_{16} \mathrm{O}_{2}$ : 192.1150. Found: 192.1156. Anal. Calcd for $\mathrm{C}_{12} \mathrm{H}_{16} \mathrm{O}_{2}$ : C, 74.97; H, 8.39. Found: C, 74.85; H, 8.42. $[\alpha]_{\mathrm{D}}{ }^{20}+21.1^{\circ}\left(c=0.75, \mathrm{CHCl}_{3}\right)$ for a sample that is $73 \%$ ee.

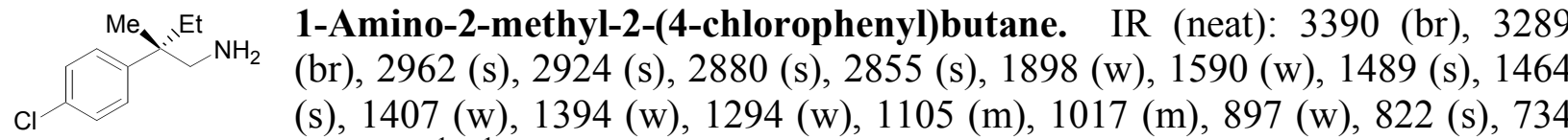
(w), $576(\mathrm{w}), 551(\mathrm{w}) \mathrm{cm}^{-1} .{ }^{1} \mathrm{H} \mathrm{NMR}\left(\mathrm{CDCl}_{3}, 400 \mathrm{MHz}\right): \delta$ 7.31-7.27 (2H, m, ArH), 7.24-7.22 
$(2 \mathrm{H}, \mathrm{m}, \mathrm{ArH}), 2.90\left(1 \mathrm{H}, \mathrm{d}, J=13.2 \mathrm{~Hz}, \mathrm{CHHNO}_{2}\right), 2.69\left(1 \mathrm{H}, \mathrm{d}, J=13.2 \mathrm{~Hz}, \mathrm{CHHNO}_{2}\right), 1.75$ $\left(1 \mathrm{H}, \mathrm{dq}, J=14.4,7.2 \mathrm{~Hz}, \mathrm{CHHCH}_{3}\right), 1.52\left(1 \mathrm{H}, \mathrm{dq}, J=14.4,7.2 \mathrm{~Hz}, \mathrm{CHHCH}_{3}\right), 1.28(3 \mathrm{H}, \mathrm{s}$, $\left.\mathrm{CCH}_{3}\right), 0.87\left(2 \mathrm{H}, \mathrm{br}, \mathrm{NH}_{2}\right), 0.70\left(3 \mathrm{H}, \mathrm{t}, J=7.2 \mathrm{~Hz}, \mathrm{CH}_{2} \mathrm{CH}_{3}\right) .{ }^{13} \mathrm{C} \mathrm{NMR}\left(\mathrm{CDCl}_{3}, 100 \mathrm{MHz}\right): \delta$ 144.3, 131.7, 128.5, 128.4, 54.0, 43.4, 32.6, 21.4, 8.5. HRMS Calcd for $\mathrm{C}_{11} \mathrm{H}_{16} \mathrm{ClN}$ : 197.0971. Found: 197.0970. 